CRYSTALLOGRAPHIC COMMUNICATIONS

ISSN 2056-9890

Received 3 June 2020

Accepted 19 June 2020

Edited by M. Weil, Vienna University of Technology, Austria

Keywords: crystal structure; manganese(I) complex; terpyridyl ligand; distinct coordination mode; disorder.

CCDC references: 2010792; 2010791

Supporting information: this article has supporting information at journals.iucr.org/e
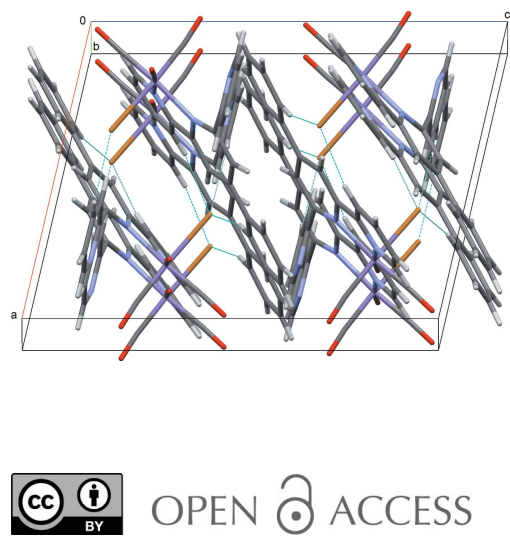

\section{Selective synthesis and crystal structures of manganese(I) complexes with a bi- or tridentate terpyridine ligand}

\author{
Kosei Wadayama, ${ }^{a}$ Tsugiko Takase $^{\mathrm{b}}$ and Dai Oyama ${ }^{\mathrm{b} *}$ \\ ${ }^{a}$ Graduate School of Science and Engineering, Fukushima University, 1 Kanayagawa, Fukushima 960-1296, Japan, and \\ ${ }^{\mathbf{b}}$ Department of Natural Sciences and Informatics, Fukushima University, 1, Kanayagawa, Fukushima 960-1296, Japan. \\ *Correspondence e-mail: daio@sss.fukushima-u.ac.jp
}

The crystal structures of two manganese(I) complexes with a different coordination mode of the supporting ligand are reported: fac-bromidotricarbonyl(4'phenyl-2,2': $6^{\prime}, 2^{\prime \prime}$-terpyridine- $\left.\kappa^{2} N, N^{\prime}\right)$ manganese $(\mathrm{I}),\left[\mathrm{MnBr}\left(\mathrm{C}_{21} \mathrm{H}_{15} \mathrm{~N}_{3}\right)(\mathrm{CO})_{3}\right], \mathbf{I}$, and cis-bromidodicarbonyl $\left(4^{\prime}\right.$-phenyl-2,2':6, $2^{\prime \prime}$-terpyridine- $\left.\kappa^{3} N, N^{\prime}, N^{\prime \prime}\right)$ manganese(I), [ $\left.\mathrm{MnBr}\left(\mathrm{C}_{21} \mathrm{H}_{15} \mathrm{~N}_{3}\right)(\mathrm{CO})_{2}\right]$, II. In both complexes, the manganese(I) atom is coordinated by terminal carbonyl ligands, a bromide ion, and a $4^{\prime}$-phenyl$2,2^{\prime}: 6^{\prime}, 2^{\prime \prime}$-terpyridine ligand within a distorted octahedral environment. In I, the metal ion is facially coordinated by three carbonyl ligands and the terpyridine ligand binds in a bidentate fashion. The non-coordinating nitrogen atom in the terpyridine ligand is positioned on the side opposite to the bromido ligand. In II, the metal ion is coordinated by two carbonyl ligands in a cis configuration and the terpyridine ligand binds in a tridentate fashion; notably, one carbonyl and the trans bromido ligand are mutually disordered over two positions. In I, the complex molecules are linked by $\mathrm{C}-\mathrm{H} \cdots \mathrm{Br}$ hydrogen bonds. In II, aromatic $\pi-\pi$ contacts are present, as well as pairs of $\mathrm{C}-\mathrm{H} \cdots \mathrm{Br}$ and $\mathrm{C}-\mathrm{H} \cdots \mathrm{O}$ hydrogen bonds.

\section{Chemical context}

Carbonylmanganese(I) complexes with polypyridyl ligands are of particular interest as novel active molecules that are able to release $\mathrm{CO}$ in response to photoirradiation (Carrington et al., 2013; Chakraborty et al., 2014; Jimenez et al., 2015) or as electrocatalysts of $\mathrm{CO}_{2}$ reduction (Grills et al., 2018; Stanbury et al., 2017). Among these compounds, studies have concentrated mainly on tricarbonyl complexes comprising bidentate polypyridyl supporting ligands; by contrast, only few reports exist on dicarbonyl complexes bearing tridentate ligands (Compain et al., 2015; Machan \& Kubiak, 2016). In fact, even though the typically tridentate ligands $2,2^{\prime}: 6^{\prime}, 2^{\prime \prime}$-terpyridine and derivatives thereof coordinate to an $\mathrm{Mn}^{\mathrm{I}}$ ion, the majority of them bind the metal ion in a bidentate manner (Compain et al., 2014; Moya et al., 2001).

As indicated by the results of studies focusing on the comparison between carbonylmanganese complexes containing bidentate and tridentate terpyridines (Compain $e t$ al., 2015; Machan \& Kubiak, 2016), investigating the relationship between reactivity and molecular structure is a key research objective. However, comparing these two systems experimentally is difficult, particularly considering that available structural data on complexes comprising tridentate terpyridine ligands are quite scarce. 


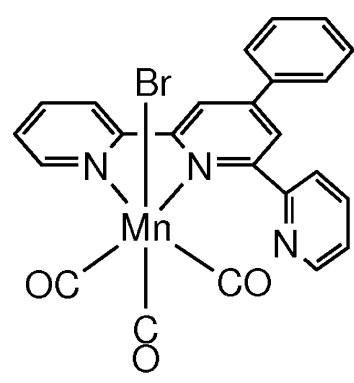

I

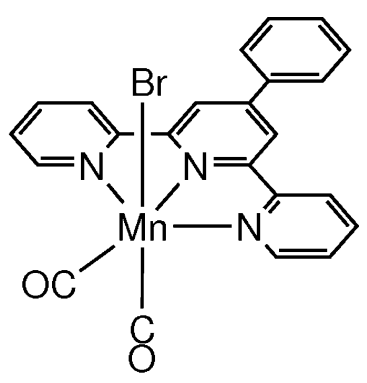

II
Herein, we report the structural characterization of complex fac $(\mathrm{CO})-\left[\mathrm{Mn}\left(\operatorname{tpyPh}-\kappa^{2} N, N^{\prime}\right)(\mathrm{CO})_{3} \mathrm{Br}\right]\left(\mathbf{I} ; \mathrm{tpyPh}=4^{\prime}-\right.$

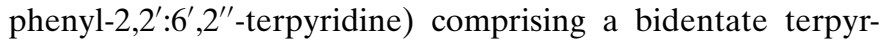
idine-based ligand, which has been synthesized by Moya et al. (2001), and the synthesis and characterization of the corresponding complex $\operatorname{cis}(\mathrm{CO})-\left[\mathrm{Mn}\left(\mathrm{tpyPh}-\kappa^{3} N, N^{\prime}, N^{\prime \prime}\right)(\mathrm{CO})_{2} \mathrm{Br}\right]$ (II), whereby the same terpyridine-based ligand is tridentate.

\section{Structural commentary}

The molecular structures of compounds I and II are displayed in Figs. 1 and 2, respectively. Although $\mathbf{I}$ was prepared by Moya et al. (2001), its structure has not previously been determined. In I and II, the manganese(I) atoms exhibit distorted octahedral coordination environments, similar to those reported for other structurally related complexes (Compain et al., 2014, 2015). In I, the fac configuration of the three CO ligands around the central manganese(I) atom is in agreement with the IR data of the complex and similar to those previously reported for complexes of this type (Compain et al., 2014, 2015). As can be evinced from Fig. 1, the terpyridine ligand exhibits a bidentate coordination with respect to the central $\mathrm{Mn}^{\mathrm{I}}$ atom, so that one of the outer

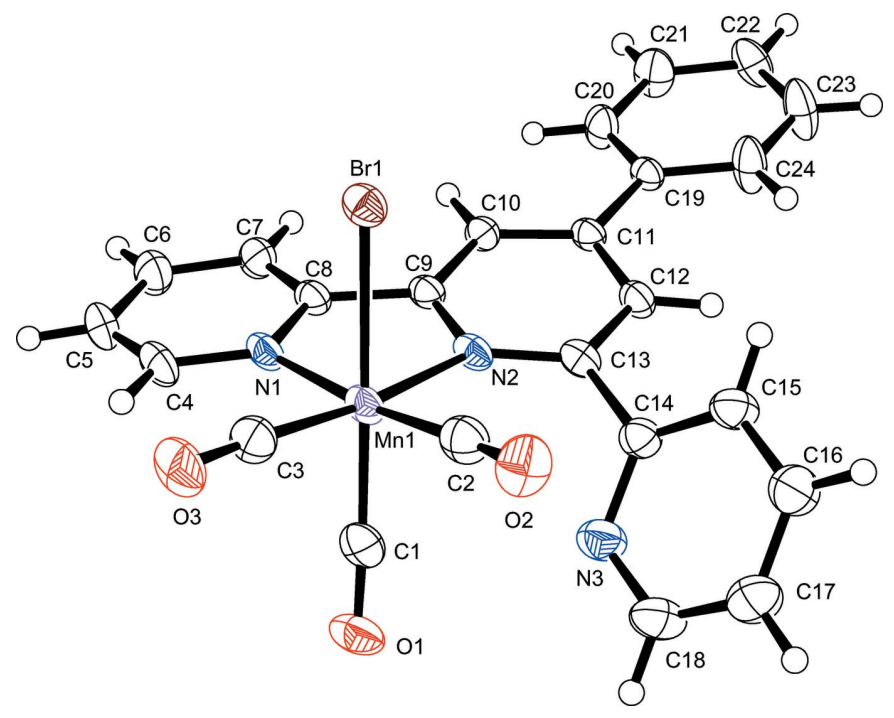

Figure 1

The molecular structure of compound I, with atom labeling and displacement ellipsoids drawn at the $50 \%$ probability level. pyridyl rings remains outside the coordination sphere. The corresponding non-coordinating $\mathrm{N}$ atom, N3, is positioned on the side opposite to the $\mathrm{Br}$ atom. As a result, the torsion angle between the coordinating and non-coordinating pyridyl rings in I $(\mathrm{N} 2-\mathrm{C} 13-\mathrm{C} 14-\mathrm{N} 3)$ is much smaller [47.9 (3) $)^{\circ}$ than those reported for related $\mathrm{Mn}^{\mathrm{I}}$ complexes with bidentate terpyridine derivatives (Compain et al., 2014, 2015). The noncoordinating $\mathrm{N}$ atom is positioned in proximity of the equatorial carbonyl ligand $(\mathrm{C} 2 \equiv \mathrm{O} 2)$, with a short value for the interatomic distance between C2 and N3 [2.900 (4) A]]. Since this distance is considerably shorter than the sum of the two atoms' van der Waals radii (3.25 A; Bondi, 1964), evidence suggests that an interaction exists between the free pyridine and the adjacent $\mathrm{CO}$ ligand. This interaction may explain the observation that the $\mathrm{Mn} 1-\mathrm{C} 2$ distance $[1.840$ (3) $\AA$ ] is longer than the other two corresponding distances in $\mathbf{I}[\mathrm{Mn} 1-\mathrm{C} 1=$ 1.805 (3) and $\mathrm{Mn} 1-\mathrm{C} 3=1.796$ (3) ̊].

The crystal structures of $\mathrm{Mn}^{\mathrm{I}}$ dicarbonyl complexes with tridentate terpyridines have very rarely been reported (Compain et al., 2015), because of the instability in solution of compounds of this type. In II, the carbonyl ligands are in cis configuration, again in accordance with IR data. Differently from I, in II the $\mathrm{Mn}^{\mathrm{I}}$ ion is coordinated by a tridentate terpyridyl ligand, as well as two $\mathrm{CO}$ ligands and a $\mathrm{Br}^{-}$ion. Only the central $\mathrm{Mn}-\mathrm{N} 2$ bond is slightly shortened (by $\sim 0.05 \AA$ ) as a result of geometric constraints. In contrast to $\mathbf{I}$, where no disorder is observed, in II one of the CO ligands $(\mathrm{C} 2 \equiv \mathrm{O} 2)$ and the $\mathrm{Br}^{-}$ligand are mutually disordered over two positions. The dihedral angle between the phenyl pendant and the central pyridyl ring in II is slightly larger than the corresponding angle in I. Specifically, the C10-C11-C19C20 torsion angle has a value of $-19.3(5)^{\circ}$ in II and $-9.9(4)^{\circ}$ in $\mathbf{I}$, but both values indicate an essential quasi-coplanarity. Notably, the extended conjugation made possible by the mentioned quasi-planarity may contribute to an increased stability of these compounds.

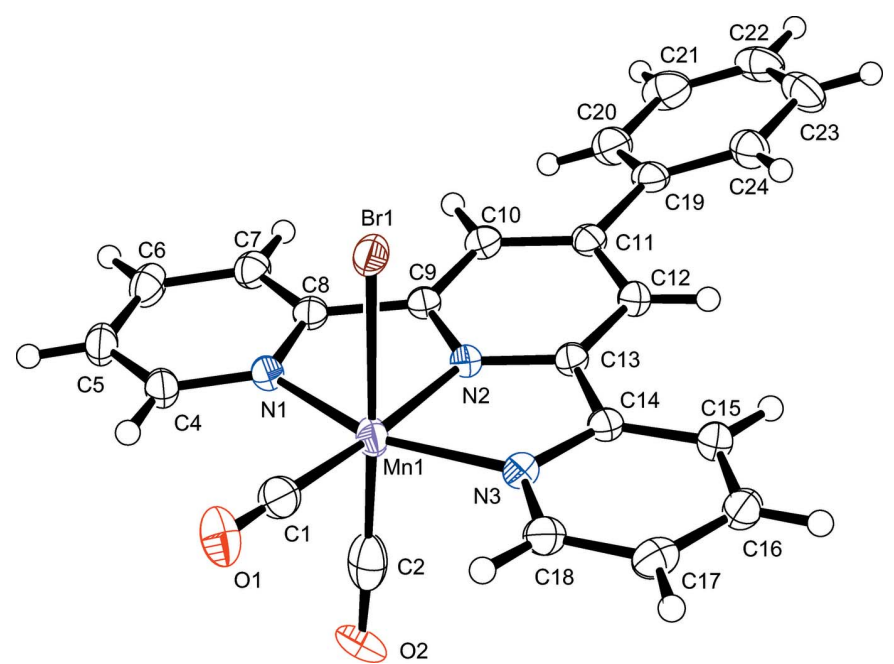

Figure 2

The molecular structure of compound II, with atom labeling and displacement ellipsoids drawn at the $50 \%$ probability level. Only the major components $(\mathrm{Br} 1 / \mathrm{C} 2 \equiv \mathrm{O} 2)$ of the disordered groups are shown. 
Table 1

Hydrogen-bond geometry $\left(\AA,^{\circ}\right)$ for $\mathbf{I}$.

\begin{tabular}{lllll}
\hline$D-\mathrm{H} \cdots A$ & $D-\mathrm{H}$ & $\mathrm{H} \cdots A$ & $D \cdots A$ & $D-\mathrm{H} \cdots A$ \\
\hline $\mathrm{C} 7-\mathrm{H} 4 \cdots \mathrm{Br} 1^{\mathrm{i}}$ & 0.95 & 2.83 & $3.754(3)$ & 165 \\
$\mathrm{C} 16-\mathrm{H} 8 \cdots \mathrm{Br}{ }^{\mathrm{ii}}$ & 0.95 & 2.88 & $3.612(4)$ & 135 \\
$\mathrm{C} 20-\mathrm{H} 11 \cdots \mathrm{Br} 1^{\mathrm{i}}$ & 0.95 & 2.92 & $3.844(2)$ & 163 \\
\hline
\end{tabular}

Symmetry codes: (i) $-x+1, y-\frac{1}{2},-z+\frac{3}{2}$; (ii) $-x+1, y+\frac{1}{2},-z+\frac{3}{2}$.

\section{Supramolecular features}

In the crystal structure of $\mathbf{I}$, complex molecules display three kinds of $\mathrm{C}-\mathrm{H} \cdots \mathrm{Br}$ hydrogen bonds (i.e., between the $\mathrm{Br}^{-}$ ligand and the $\mathrm{C}-\mathrm{H}$ groups in the coordinating pyridyl ring, the free pyridyl ring, and the phenyl pendant), forming a three-dimensional supramolecular structure (Table 1 and Fig. 3).

In the crystal structure of II, weak $\mathrm{C}-\mathrm{H} \cdots \mathrm{Br}$ and $\mathrm{C}-$ $\mathrm{H}$. . O hydrogen bonding interactions (Table 2) exist between the terpyridyl ligand and the disordered $\mathrm{CO} / \mathrm{Br}$ ligands. Additional $\pi-\pi$ interactions $\left[C g 3 \cdots C g 2^{\text {iv }}=4.000\right.$ (2) and $C g 1 \cdots C g 1^{\mathrm{i}}=4.128$ (3) $\AA$; $C g 1, C g 2$ and $C g 3$ are the centroids of the N1/C4-C8, N2/C9-C13 and N3/C14-C18 rings, respectively; symmetry codes: (i) $1-x,-y, 2-z$; (iv) $\left.x,-y+\frac{1}{2}, z-\frac{1}{2}\right]$ consolidate the crystal packing. These interactions lead to the formation of a three-dimensional network structure (Fig. 4).

\section{Database survey}

With respect to manganese(I) complexes with a tridentate terpyridine derivative ligand of the form cis(CO)$\left[\mathrm{Mn}(\operatorname{tpy} R)(\mathrm{CO})_{2} \mathrm{Br}\right]$, only a single structure, whereby $R=p$ tolyl, has been reported (Compain et al., 2015). In contrast, some structures of bidentate terpyridine derivative-coordinated manganese(I) complexes have been reported by Compain et al. (2014, 2015).

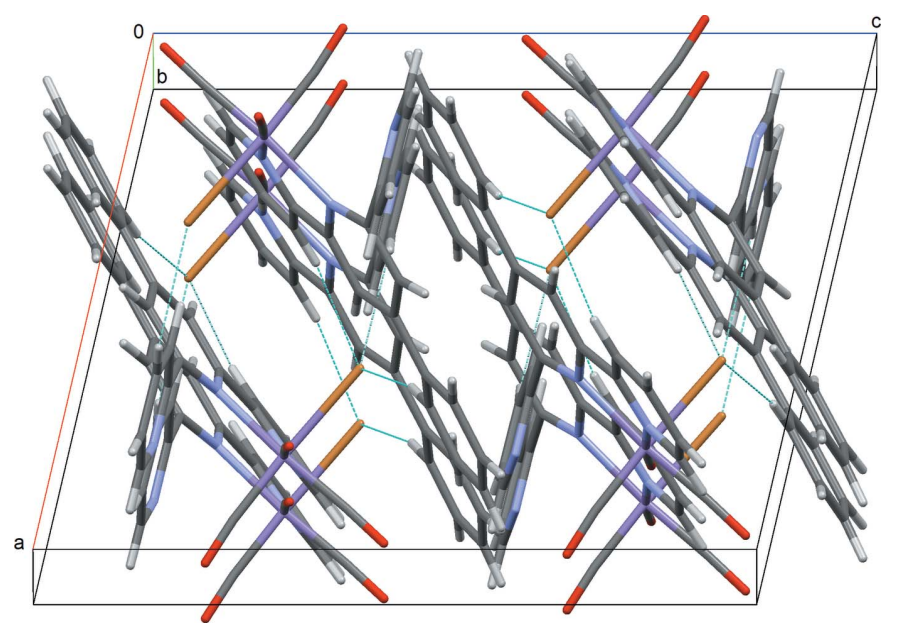

Figure 3

The crystal packing of compound I with $\mathrm{C}-\mathrm{H} \cdots \mathrm{Br}$ hydrogen bonds shown as dashed lines.
Table 2

Hydrogen-bond geometry $\left(\AA,^{\circ}\right)$ for II.

\begin{tabular}{lllll}
\hline$D-\mathrm{H} \cdots A$ & $D-\mathrm{H}$ & $\mathrm{H} \cdots A$ & $D \cdots A$ & $D-\mathrm{H} \cdots A$ \\
\hline $\mathrm{C} 5-\mathrm{H} 2 \cdots \mathrm{Br} 1^{\mathrm{i}}$ & 0.95 & 2.84 & $3.528(4)$ & 130 \\
$\mathrm{C} 7-\mathrm{H} 4 \cdots \mathrm{Br} 1^{1 i}$ & 0.95 & 2.86 & $3.771(4)$ & 162 \\
$\mathrm{C} 12-\mathrm{H} 6 \cdots \mathrm{Br}^{\mathrm{iii}}$ & 0.95 & 2.75 & $3.688(7)$ & 171 \\
$\mathrm{C} 12-\mathrm{H} 6 \cdots \mathrm{O} 2^{\mathrm{iii}}$ & 0.95 & 2.55 & $3.491(7)$ & 173 \\
$\mathrm{C} 15-\mathrm{H} 7 \cdots \mathrm{Br} 2^{\mathrm{iii}}$ & 0.95 & 2.81 & $3.759(7)$ & 175 \\
$\mathrm{C} 15-\mathrm{H} 7 \cdots \mathrm{O} 2^{\mathrm{iii}}$ & 0.95 & 2.50 & $3.447(7)$ & 172 \\
$\mathrm{C} 16-\mathrm{H} 8 \cdots \mathrm{Br}^{\mathrm{iv}}$ & 0.95 & 2.52 & $3.286(7)$ & 138 \\
$\mathrm{C} 16-\mathrm{H} 8 \cdots 2^{\mathrm{iv}}$ & 0.95 & 2.57 & $3.363(7)$ & 141 \\
$\mathrm{C} 20-\mathrm{H} 11 \cdots \mathrm{Br} 1^{\mathrm{ii}}$ & 0.95 & 2.81 & $3.743(4)$ & 168 \\
$\mathrm{C} 20-\mathrm{H} 11 \cdots \mathrm{O} 3^{\mathrm{ii}}$ & 0.95 & 2.55 & $3.446(18)$ & 158 \\
$\mathrm{C} 24-\mathrm{H} 15 \cdots \mathrm{Br} 2^{\mathrm{iii}}$ & 0.95 & 2.84 & $3.611(7)$ & 139 \\
\hline
\end{tabular}

Symmetry codes: (i) $-x+1,-y,-z+2$; (ii) $x,-y+\frac{1}{2}, z+\frac{1}{2}$; (iii) $-x, y+\frac{1}{2},-z+\frac{3}{2}$; (iv) $x,-y+\frac{1}{2}, z-\frac{1}{2}$.

\section{Synthesis and crystallization}

All the manganese(I) complexes were handled and stored in the dark to minimize exposure to light. Compound I was synthesized as described by Moya et al. (2001). The compound thus obtained proved to be analytically and spectroscopically pure (as determined by microanalysis, IR, UV-vis, and ${ }^{1} \mathrm{H}$ NMR data). Crystals suitable for use in X-ray diffraction experiments were grown by vapor diffusion of diethyl ether into an acetone solution of $\mathbf{I}$.

For the synthesis of compound II, bromidopentacarbonylmanganese(I) (30 mg, $0.11 \mathrm{mmol}$ ) and $4^{\prime}$-phenyl-2,2': $6^{\prime}, 2^{\prime \prime}$ terpyridine $(31 \mathrm{mg}, 0.10 \mathrm{mmol})$ were dissolved in an acetonewater mixture $(20 / 30 \mathrm{ml})$. The solution thus obtained was refluxed for $24 \mathrm{~h}$; the solvent was then evaporated under reduced pressure, and the resulting solid was placed in diethyl ether $(50 \mathrm{ml})$; the resulting mixture was stirred for $30 \mathrm{~min}$ to remove the starting materials and subsequently filtered; the isolated residue was washed with diethyl ether to obtain a yield for the desired complex of $43 \mathrm{mg}(86 \%)$. Single crystals suitable for X-ray diffraction experiments were grown by slow vapor diffusion of $n$-hexane into an acetone solution of II. FTIR $v_{\mathrm{CO}}\left(\mathrm{KBr}\right.$ pellet): $1916(s), 1838(s) \mathrm{cm}^{-1}$.

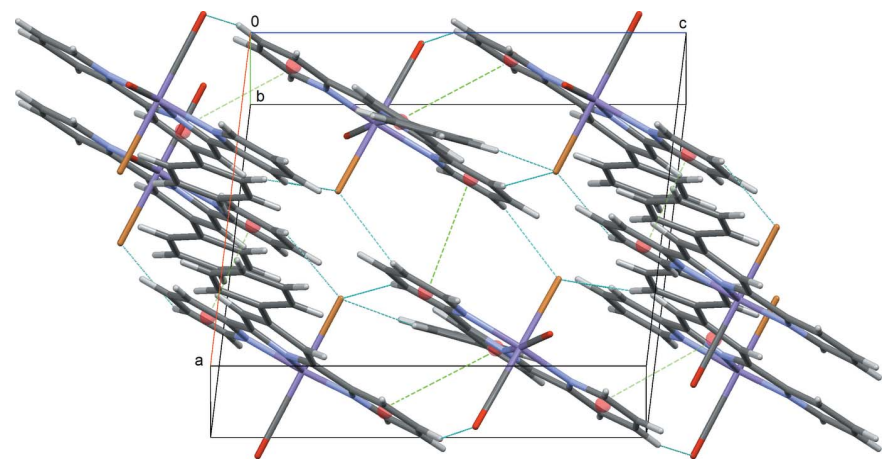

Figure 4

The crystal packing of compound II with $\mathrm{C}-\mathrm{H} \cdots \mathrm{Br}$ and $\mathrm{C}-\mathrm{H} \cdots \mathrm{O}$ hydrogen bonds (blue) and $\pi-\pi$ contacts (green) shown as dashed lines; ring centroids are shown as red spheres. 
Table 3

Experimental details.

I

Crystal data

Chemical formula

$M_{\mathrm{r}}$

Crystal system, space group

Temperature (K)

$a, b, c(\AA)$

$\beta\left({ }^{\circ}\right)$

$V\left(\AA^{3}\right)$

Z

Radiation type

$\mu\left(\mathrm{mm}^{-1}\right)$

Crystal size (mm)

Data collection

Diffractometer

Absorption correction

$T_{\min }, T_{\max }$

No. of measured, independent and

observed $\left[F^{2}>2.0 \sigma\left(F^{2}\right)\right]$ reflections

$R_{\text {int }}$

$(\sin \theta / \lambda)_{\max }\left(\AA^{-1}\right)$

Refinement

$R\left[F^{2}>2 \sigma\left(F^{2}\right)\right], w R\left(F^{2}\right), S$

No. of reflections

No. of parameters

No. of restraints

$\mathrm{H}$-atom treatment

$\Delta \rho_{\max }, \Delta \rho_{\min }\left(\mathrm{e} \AA^{-3}\right)$
II
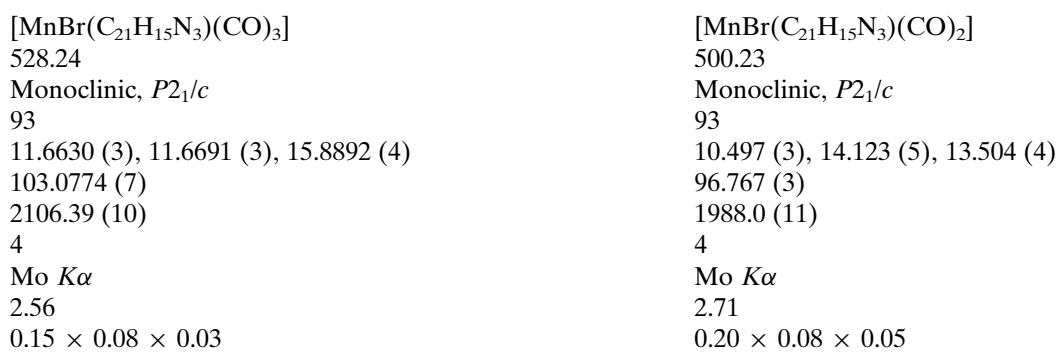

Rigaku Saturn70

Multi-scan (REQAB, Rigaku, 1998)

$0.774,0.926$

$21455,4813,4253$

0.030

0.649

Rigaku Saturn70

Multi-scan (REQAB, Rigaku, 1998) $0.795,0.873$

19872, 4518, 4016

0.028

0.649

$0.035,0.092,1.06$

4813

289

0

$\mathrm{H}$-atom parameters constrained

$0.96,-0.32$
$0.046,0.096,1.27$

4518

289

3

$\mathrm{H}$-atom parameters constrained $0.83,-0.80$

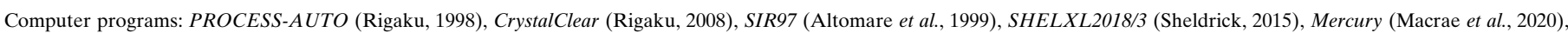
ORTEP-3 for Windows (Farrugia, 2012), CrystalStructure (Rigaku, 2019), PLATON (Spek, 2020) and publCIF (Westrip, 2010).

\section{Refinement}

Crystal data, data collection, and structure refinement details are summarized in Table 3 . All hydrogen atoms were placed at calculated positions $(\mathrm{C}-\mathrm{H}=0.95 \AA)$ and refined using a riding model with $U_{\text {iso }}(\mathrm{H})=1.2 U_{\text {eq }}(\mathrm{C})$. In compound II, the $\mathrm{CO}$ group and the $\mathrm{Br}$ atom trans to it were refined as being disordered over two sets of sites, $(\mathrm{Br} 1 / \mathrm{C} 2 \equiv \mathrm{O} 2)$ and $(\mathrm{Br} 2 /$ $\mathrm{C} 3 \equiv \mathrm{O} 3)$, respectively, with an occupancy ratio of 0.807 (2): 0.193 (2).

\section{Funding information}

Funding for this research was provided by: Japan Society for the Promotion of Science (grant No. JP17K05799).

\section{References}

Altomare, A., Burla, M. C., Camalli, M., Cascarano, G. L., Giacovazzo, C., Guagliardi, A., Moliterni, A. G. G., Polidori, G. \& Spagna, R. (1999). J. Appl. Cryst. 32, 115-119.

Bondi, A. (1964). J. Phys. Chem. 68, 441-451.

Carrington, S. J., Chakraborty, I. \& Mascharak, P. K. (2013). Chem. Commun. 49, 11254-11256.
Chakraborty, I., Carrington, S. J. \& Mascharak, P. K. (2014). ChemMedChem, 9, 1266-1274.

Compain, J.-D., Bourrez, M., Haukka, M., Deronzier, A. \& ChardonNoblat, S. (2014). Chem. Commun. 50, 2539-2542.

Compain, J.-D., Stanbury, M., Trejo, M. \& Chardon-Noblat, S. (2015). Eur. J. Inorg. Chem. pp. 5757-5766.

Farrugia, L. J. (2012). J. Appl. Cryst. 45, 849-854.

Grills, D. C., Ertem, M. Z., McKinnon, M., Ngo, K. T. \& Rochford, J. (2018). Coord. Chem. Rev. 374, 173-217.

Jimenez, J., Chakraborty, I. \& Mascharak, P. K. (2015). Eur. J. Inorg. Chem. pp. 5021-5026.

Machan, C. W. \& Kubiak, C. P. (2016). Dalton Trans. 45, 17179-17186.

Macrae, C. F., Sovago, I., Cottrell, S. J., Galek, P. T. A., McCabe, P., Pidcock, E., Platings, M., Shields, G. P., Stevens, J. S., Towler, M. \& Wood, P. A. (2020). J. Appl. Cryst. 53, 226-235.

Moya, S. A., Pastene, R., Le Bozec, H., Baricelli, P. J., Pardey, A. J. \& Gimeno, J. (2001). Inorg. Chim. Acta, 312, 7-14.

Rigaku (1998). REQAB and PROCESS-AUTO. Rigaku Corporation, Tokyo, Japan.

Rigaku (2008). CrystalClear. Rigaku Corporation, Tokyo, Japan.

Rigaku (2019). CrystalStructure. Rigaku Corporation, Tokyo, Japan.

Sheldrick, G. M. (2015). Acta Cryst. C71, 3-8.

Spek, A. L. (2020). Acta Cryst. E76, 1-11.

Stanbury, M., Compain, J.-D., Trejo, M., Smith, P., Gouré, E. \& Chardon-Noblat, S. (2017). Electrochim. Acta, 240, 288-299.

Westrip, S. P. (2010). J. Appl. Cryst. 43, 920-925. 


\section{supporting information}

Acta Cryst. (2020). E76, 1139-1142［https://doi.org/10.1107/S2056989020008178]

\section{Selective synthesis and crystal structures of manganese(I) complexes with a bi- or tridentate terpyridine ligand}

\section{Kosei Wadayama, Tsugiko Takase and Dai Oyama}

Computing details

Data collection: PROCESS-AUTO (Rigaku, 1998) for (I); CrystalClear (Rigaku, 2008) for (II). Cell refinement: PROCESS-AUTO (Rigaku, 1998) for (I); CrystalClear (Rigaku, 2008) for (II). Data reduction: PROCESS-AUTO (Rigaku, 1998) for (I); CrystalClear (Rigaku, 2008) for (II). For both structures, program(s) used to solve structure: SIR97 (Altomare et al., 1999); program(s) used to refine structure: SHELXL2018/3 (Sheldrick, 2015); molecular graphics: Mercury (Macrae et al., 2020), ORTEP-3 for Windows (Farrugia, 2012); software used to prepare material for publication: CrystalStructure (Rigaku, 2019), PLATON (Spek, 2020), publCIF (Westrip, 2010).

fac-Bromidotricarbonyl(4'-phenyl-2,2':6', 2"'-terpyridine- $\left.\kappa^{2} N, N^{\prime}\right)$ manganese(I) (I)

\section{Crystal data}

$\left[\mathrm{MnBr}\left(\mathrm{C}_{21} \mathrm{H}_{15} \mathrm{~N}_{3}\right)(\mathrm{CO})_{3}\right]$

$M_{r}=528.24$

Monoclinic, $P 2_{1} / c$

$a=11.6630(3) \AA$

$b=11.6691(3) \AA$

$c=15.8892(4) \AA$

$\beta=103.0774(7)^{\circ}$

$V=2106.39(10) \AA^{3}$

$Z=4$

\section{Data collection}

Rigaku Saturn70 diffractometer

Detector resolution: 7.143 pixels $\mathrm{mm}^{-1}$ $\omega$ scans

Absorption correction: multi-scan (REQAB, Rigaku, 1998)

$T_{\min }=0.774, T_{\max }=0.926$

21455 measured reflections

\section{Refinement}

Refinement on $F^{2}$

$R\left[F^{2}>2 \sigma\left(F^{2}\right)\right]=0.035$

$w R\left(F^{2}\right)=0.092$

$S=1.06$

4813 reflections

289 parameters

0 restraints
$F(000)=1056.00$

$D_{\mathrm{x}}=1.666 \mathrm{Mg} \mathrm{m}^{-3}$

Mo $K \alpha$ radiation, $\lambda=0.71075 \AA$

Cell parameters from 18973 reflections

$\theta=3.0-27.5^{\circ}$

$\mu=2.56 \mathrm{~mm}^{-1}$

$T=93 \mathrm{~K}$

Platelet, orange

$0.15 \times 0.08 \times 0.03 \mathrm{~mm}$

4813 independent reflections

4253 reflections with $F^{2}>2.0 \sigma\left(F^{2}\right)$

$R_{\text {int }}=0.030$

$\theta_{\max }=27.5^{\circ}, \theta_{\min }=3.0^{\circ}$

$h=-15 \rightarrow 15$

$k=-15 \rightarrow 15$

$l=-20 \rightarrow 19$

Primary atom site location: structure-invariant direct methods

Secondary atom site location: difference Fourier map

Hydrogen site location: inferred from neighbouring sites

$\mathrm{H}$-atom parameters constrained 
$w=1 /\left[\sigma^{2}\left(F_{\mathrm{o}}^{2}\right)+(0.0416 P)^{2}+2.6282 P\right]$

where $P=\left(F_{\mathrm{o}}^{2}+2 F_{\mathrm{c}}{ }^{2}\right) / 3$

$(\Delta / \sigma)_{\max }=0.001$

$$
\Delta \rho_{\max }=0.96 \mathrm{e} \AA^{-3}
$$

Special details

Refinement. Refinement was performed using all reflections. The weighted R-factor (wR) and goodness of fit (S) are based on $\mathrm{F}^{2}$. R-factor (gt) are based on $\mathrm{F}$. The threshold expression of $\mathrm{F}^{2}>2.0 \operatorname{sigma}\left(\mathrm{F}^{2}\right)$ is used only for calculating Rfactor $(\mathrm{gt})$.

Fractional atomic coordinates and isotropic or equivalent isotropic displacement parameters $\left(\AA^{2}\right)$

\begin{tabular}{|c|c|c|c|c|}
\hline & $x$ & $y$ & $z$ & $U_{\text {iso }} * / U_{\text {eq }}$ \\
\hline $\mathrm{Br} 1$ & $0.61476(2)$ & $0.17999(2)$ & $0.88742(2)$ & $0.02812(9)$ \\
\hline Mn1 & $0.78248(3)$ & $0.17015(3)$ & $0.81054(2)$ & $0.02513(10)$ \\
\hline $\mathrm{O} 1$ & 0.99149 (17) & $0.17222(19)$ & $0.73649(14)$ & $0.0400(5)$ \\
\hline $\mathrm{O} 2$ & $0.7925(2)$ & $0.42515(18)$ & $0.81993(14)$ & $0.0444(5)$ \\
\hline $\mathrm{O} 3$ & 0.95375 (19) & $0.1798(2)$ & $0.97692(14)$ & $0.0457(5)$ \\
\hline N1 & $0.76212(17)$ & -0.00325 (19) & $0.81015(13)$ & $0.0252(4)$ \\
\hline N2 & $0.65411(17)$ & 0.14582 (19) & $0.69692(13)$ & $0.0238(4)$ \\
\hline N3 & $0.7794(2)$ & $0.3305(2)$ & $0.62983(17)$ & $0.0359(5)$ \\
\hline $\mathrm{C} 1$ & $0.9075(2)$ & $0.1690(2)$ & $0.76117(18)$ & $0.0320(6)$ \\
\hline $\mathrm{C} 2$ & 0.7848 & 0.3278 & $0.81319(19)$ & $0.0354(6)$ \\
\hline $\mathrm{C} 3$ & $0.8852(2)$ & $0.1758(2)$ & $0.91343(18)$ & $0.0327(6)$ \\
\hline $\mathrm{C} 4$ & $0.8291(2)$ & $-0.0776(2)$ & $0.86473(17)$ & $0.0313(6)$ \\
\hline H1 & 0.893046 & -0.048384 & 0.907298 & $0.038^{*}$ \\
\hline $\mathrm{C} 5$ & $0.8095(2)$ & $-0.1941(3)$ & 0.86198 (19) & $0.0358(6)$ \\
\hline $\mathrm{H} 2$ & 0.860275 & -0.243958 & 0.900658 & $0.043^{*}$ \\
\hline C6 & $0.7144(2)$ & $-0.2373(3)$ & 0.80188 (19) & $0.0350(6)$ \\
\hline H3 & 0.698453 & -0.317169 & 0.798723 & $0.042 *$ \\
\hline $\mathrm{C} 7$ & $0.6431(2)$ & $-0.1615(2)$ & $0.74643(18)$ & $0.0313(6)$ \\
\hline $\mathrm{H} 4$ & 0.576614 & -0.188952 & 0.705236 & $0.038 *$ \\
\hline $\mathrm{C} 8$ & $0.6693(2)$ & $-0.0454(2)$ & $0.75134(15)$ & $0.0247(5)$ \\
\hline $\mathrm{C} 9$ & $0.6038(2)$ & $0.0406(2)$ & $0.69157(15)$ & $0.0232(5)$ \\
\hline $\mathrm{C} 10$ & $0.4996(2)$ & $0.0143(2)$ & $0.63271(15)$ & $0.0232(5)$ \\
\hline H5 & 0.466483 & -0.060220 & 0.632112 & $0.028 *$ \\
\hline C11 & 0.44359 (19) & $0.0971(2)$ & $0.57461(15)$ & $0.0214(5)$ \\
\hline C12 & $0.5003(2)$ & $0.2023(2)$ & $0.57681(16)$ & $0.0248(5)$ \\
\hline H6 & 0.467288 & 0.260028 & 0.536409 & $0.030 *$ \\
\hline C13 & $0.6042(2)$ & $0.2244(2)$ & $0.63698(16)$ & $0.0254(5)$ \\
\hline C14 & $0.6644(2)$ & $0.3362(2)$ & $0.63063(17)$ & $0.0280(5)$ \\
\hline C15 & $0.6033(2)$ & $0.4378(2)$ & 0.62349 (19) & $0.0326(6)$ \\
\hline $\mathrm{H} 7$ & 0.522062 & 0.438538 & 0.624227 & $0.039 *$ \\
\hline C16 & $0.6619(3)$ & 0.5393 & $0.6152(2)$ & $0.0418(7)$ \\
\hline H8 & 0.621545 & 0.610530 & 0.610537 & $0.050 *$ \\
\hline $\mathrm{C} 17$ & $0.7798(3)$ & 0.5349 & $0.6138(2)$ & $0.0446(7)$ \\
\hline H9 & 0.822144 & 0.602872 & 0.607979 & $0.054 *$ \\
\hline C18 & 0.8345 & $0.4297(3)$ & $0.6212(2)$ & $0.0403(7)$ \\
\hline $\mathrm{H} 10$ & 0.915543 & 0.426919 & 0.620071 & $0.048 *$ \\
\hline C19 & $0.3301(2)$ & $0.0749(2)$ & $0.51219(15)$ & $0.0215(5)$ \\
\hline
\end{tabular}




\begin{tabular}{lllll}
$\mathrm{C} 20$ & $0.2826(2)$ & $-0.0347(2)$ & $0.49944(17)$ & $0.0316(6)$ \\
$\mathrm{H} 11$ & 0.324220 & -0.097276 & 0.530401 & $0.038^{*}$ \\
$\mathrm{C} 21$ & $0.1756(3)$ & $-0.0542(3)$ & $0.44231(19)$ & $0.0389(7)$ \\
$\mathrm{H} 12$ & 0.144475 & -0.129640 & 0.434142 & $0.047^{*}$ \\
$\mathrm{C} 22$ & $0.1145(2)$ & $0.0361(3)$ & $0.39740(19)$ & $0.0373(6)$ \\
$\mathrm{H} 13$ & 0.039539 & 0.023966 & 0.359983 & $0.045^{*}$ \\
$\mathrm{C} 23$ & $0.1626(3)$ & $0.1429(3)$ & $0.4072(2)$ & $0.0453(8)$ \\
$\mathrm{H} 14$ & 0.122516 & 0.204709 & 0.374184 & $0.054^{*}$ \\
$\mathrm{C} 24$ & $0.2688(3)$ & $0.1626(2)$ & $0.4644(2)$ & $0.0402(7)$ \\
$\mathrm{H} 15$ & 0.300073 & 0.238042 & 0.470790 & $0.048^{*}$ \\
\hline
\end{tabular}

Atomic displacement parameters $\left(\AA^{2}\right)$

\begin{tabular}{|c|c|c|c|c|c|c|}
\hline & $U^{11}$ & $U^{22}$ & $U^{33}$ & $U^{12}$ & $U^{13}$ & $U^{23}$ \\
\hline $\mathrm{Br} 1$ & $0.02474(13)$ & $0.03228(14)$ & $0.02610(14)$ & $0.00130(9)$ & $0.00315(10)$ & $-0.00370(10)$ \\
\hline Mn1 & $0.01744(18)$ & $0.0327(2)$ & $0.0221(2)$ & $-0.00087(14)$ & $-0.00207(14)$ & $-0.00159(15)$ \\
\hline $\mathrm{O} 1$ & $0.0209(9)$ & $0.0603(14)$ & $0.0372(11)$ & $-0.0046(9)$ & $0.0033(8)$ & $-0.0054(10)$ \\
\hline $\mathrm{O} 2$ & $0.0517(13)$ & $0.0352(11)$ & $0.0424(12)$ & $-0.0087(10)$ & $0.0023(10)$ & $-0.0043(9)$ \\
\hline $\mathrm{O} 3$ & $0.0325(11)$ & $0.0571(14)$ & $0.0377(12)$ & $-0.0050(9)$ & $-0.0125(9)$ & $-0.0002(10)$ \\
\hline N1 & $0.0163(9)$ & $0.0359(11)$ & $0.0211(10)$ & $0.0032(8)$ & $-0.0007(8)$ & $-0.0008(8)$ \\
\hline $\mathrm{N} 2$ & $0.0168(9)$ & $0.0321(11)$ & $0.0209(10)$ & $0.0015(8)$ & $0.0005(8)$ & $-0.0036(8)$ \\
\hline N3 & $0.0263(11)$ & $0.0384(13)$ & $0.0427(14)$ & $-0.0042(9)$ & $0.0072(10)$ & $-0.0009(10)$ \\
\hline $\mathrm{C} 1$ & $0.0241(13)$ & $0.0399(15)$ & $0.0271(13)$ & $-0.0049(10)$ & $-0.0045(10)$ & $-0.0031(11)$ \\
\hline $\mathrm{C} 2$ & $0.0300(14)$ & $0.0417(16)$ & $0.0323(15)$ & $-0.0015(11)$ & $0.0028(11)$ & $-0.0017(12)$ \\
\hline $\mathrm{C} 3$ & $0.0303(14)$ & $0.0343(14)$ & $0.0312(14)$ & $-0.0039(11)$ & $0.0019(11)$ & $-0.0009(11)$ \\
\hline $\mathrm{C} 4$ & $0.0212(12)$ & $0.0407(15)$ & $0.0271(13)$ & $0.0043(10)$ & $-0.0049(10)$ & $-0.0010(11)$ \\
\hline $\mathrm{C} 5$ & $0.0266(13)$ & $0.0432(16)$ & $0.0323(15)$ & $0.0071(11)$ & $-0.0041(11)$ & $0.0091(12)$ \\
\hline C6 & $0.0300(13)$ & $0.0368(15)$ & $0.0345(15)$ & $-0.0005(11)$ & $-0.0005(11)$ & $0.0060(12)$ \\
\hline $\mathrm{C} 7$ & $0.0252(12)$ & $0.0385(15)$ & $0.0266(13)$ & $-0.0036(10)$ & $-0.0017(10)$ & $0.0053(11)$ \\
\hline $\mathrm{C} 8$ & $0.0178(11)$ & $0.0354(13)$ & $0.0198(11)$ & $0.0014(9)$ & $0.0019(9)$ & $0.0013(10)$ \\
\hline C9 & $0.0178(10)$ & $0.0328(13)$ & $0.0186(11)$ & $0.0010(9)$ & $0.0031(9)$ & $0.0008(9)$ \\
\hline $\mathrm{C} 10$ & $0.0187(10)$ & $0.0280(12)$ & $0.0212(11)$ & $-0.0022(9)$ & $0.0009(9)$ & $0.0020(9)$ \\
\hline $\mathrm{C} 11$ & $0.0165(10)$ & $0.0282(12)$ & $0.0184(11)$ & $0.0000(9)$ & $0.0016(8)$ & $-0.0003(9)$ \\
\hline $\mathrm{C} 12$ & $0.0185(11)$ & $0.0276(12)$ & $0.0263(12)$ & $0.0017(9)$ & $0.0007(9)$ & $0.0011(10)$ \\
\hline $\mathrm{C} 13$ & $0.0196(11)$ & $0.0287(12)$ & $0.0267(13)$ & $0.0008(9)$ & $0.0027(10)$ & $-0.0034(10)$ \\
\hline $\mathrm{C} 14$ & $0.0246(12)$ & $0.0308(13)$ & $0.0276(13)$ & $-0.0025(10)$ & $0.0038(10)$ & $-0.0005(10)$ \\
\hline $\mathrm{C} 15$ & $0.0259(12)$ & $0.0313(13)$ & $0.0412(15)$ & $-0.0036(10)$ & $0.0089(11)$ & $-0.0030(11)$ \\
\hline $\mathrm{C} 16$ & $0.0374(15)$ & $0.0309(14)$ & $0.0568(19)$ & $-0.0007(12)$ & $0.0103(14)$ & $0.0005(13)$ \\
\hline $\mathrm{C} 17$ & $0.0380(16)$ & $0.0354(15)$ & $0.060(2)$ & $-0.0105(13)$ & $0.0105(14)$ & $0.0009(14)$ \\
\hline $\mathrm{C} 18$ & $0.0279(14)$ & $0.0428(16)$ & $0.0506(18)$ & $-0.0074(12)$ & $0.0100(13)$ & $-0.0054(14)$ \\
\hline C19 & $0.0179(10)$ & $0.0283(12)$ & $0.0172(11)$ & $0.0023(9)$ & $0.0018(9)$ & $-0.0002(9)$ \\
\hline $\mathrm{C} 20$ & $0.0300(13)$ & $0.0320(13)$ & $0.0278(13)$ & $-0.0007(10)$ & $-0.0039(11)$ & $0.0056(11)$ \\
\hline $\mathrm{C} 21$ & $0.0344(15)$ & $0.0347(15)$ & $0.0399(16)$ & $-0.0088(12)$ & $-0.0078(12)$ & $0.0015(12)$ \\
\hline $\mathrm{C} 22$ & $0.0246(13)$ & $0.0417(16)$ & $0.0368(15)$ & $0.0031(11)$ & $-0.0115(11)$ & $-0.0062(12)$ \\
\hline $\mathrm{C} 23$ & $0.0420(17)$ & $0.0332(15)$ & $0.0465(18)$ & $0.0079(13)$ & -0.0194 & $0.0018(13)$ \\
\hline $\mathrm{C} 24$ & $0.0377(16)$ & $0.0266(13)$ & $0.0432(17)$ & $-0.0041(11)$ & $-0.0182(13)$ & $0.0049(12)$ \\
\hline
\end{tabular}


Geometric parameters $\left(\AA,{ }^{\circ}\right)$

\begin{tabular}{|c|c|c|c|}
\hline $\mathrm{Br} 1-\mathrm{Mn} 1$ & $2.5325(5)$ & $\mathrm{C} 10-\mathrm{H} 5$ & 0.9500 \\
\hline $\mathrm{Mn} 1-\mathrm{C} 3$ & $1.796(3)$ & $\mathrm{C} 11-\mathrm{C} 12$ & $1.390(3)$ \\
\hline $\mathrm{Mn} 1-\mathrm{C} 1$ & $1.805(3)$ & $\mathrm{C} 11-\mathrm{C} 19$ & $1.486(3)$ \\
\hline $\mathrm{Mn} 1-\mathrm{C} 2$ & $1.840(3)$ & $\mathrm{C} 12-\mathrm{C} 13$ & $1.388(3)$ \\
\hline $\mathrm{Mn} 1-\mathrm{N} 1$ & $2.037(2)$ & $\mathrm{C} 12-\mathrm{H} 6$ & 0.9500 \\
\hline $\mathrm{Mn} 1-\mathrm{N} 2$ & $2.088(2)$ & $\mathrm{C} 13-\mathrm{C} 14$ & $1.496(3)$ \\
\hline $\mathrm{O} 1-\mathrm{C} 1$ & $1.135(4)$ & $\mathrm{C} 14-\mathrm{C} 15$ & $1.375(4)$ \\
\hline $\mathrm{O} 2-\mathrm{C} 2$ & $1.143(3)$ & $\mathrm{C} 15-\mathrm{C} 16$ & $1.388(4)$ \\
\hline $\mathrm{O} 3-\mathrm{C} 3$ & $1.138(3)$ & $\mathrm{C} 15-\mathrm{H} 7$ & 0.9500 \\
\hline $\mathrm{N} 1-\mathrm{C} 4$ & $1.345(3)$ & $\mathrm{C} 16-\mathrm{C} 17$ & $1.381(4)$ \\
\hline $\mathrm{N} 1-\mathrm{C} 8$ & $1.353(3)$ & $\mathrm{C} 16-\mathrm{H} 8$ & 0.9500 \\
\hline $\mathrm{N} 2-\mathrm{C} 9$ & $1.355(3)$ & $\mathrm{C} 17-\mathrm{C} 18$ & $1.376(4)$ \\
\hline $\mathrm{N} 2-\mathrm{C} 13$ & $1.355(3)$ & $\mathrm{C} 17-\mathrm{H} 9$ & 0.9500 \\
\hline N3-C18 & $1.346(4)$ & $\mathrm{C} 18-\mathrm{H} 10$ & 0.9500 \\
\hline N3-C14 & $1.347(3)$ & $\mathrm{C} 19-\mathrm{C} 24$ & $1.374(3)$ \\
\hline $\mathrm{C} 4-\mathrm{C} 5$ & $1.377(4)$ & $\mathrm{C} 19-\mathrm{C} 20$ & $1.391(4)$ \\
\hline $\mathrm{C} 4-\mathrm{H} 1$ & 0.9500 & $\mathrm{C} 20-\mathrm{C} 21$ & $1.386(4)$ \\
\hline $\mathrm{C} 5-\mathrm{C} 6$ & $1.384(4)$ & $\mathrm{C} 20-\mathrm{H} 11$ & 0.9500 \\
\hline $\mathrm{C} 5-\mathrm{H} 2$ & 0.9500 & $\mathrm{C} 21-\mathrm{C} 22$ & $1.377(4)$ \\
\hline $\mathrm{C} 6-\mathrm{C} 7$ & $1.385(4)$ & $\mathrm{C} 21-\mathrm{H} 12$ & 0.9500 \\
\hline $\mathrm{C} 6-\mathrm{H} 3$ & 0.9500 & $\mathrm{C} 22-\mathrm{C} 23$ & $1.361(4)$ \\
\hline $\mathrm{C} 7-\mathrm{C} 8$ & $1.387(4)$ & $\mathrm{C} 22-\mathrm{H} 13$ & 0.9500 \\
\hline $\mathrm{C} 7-\mathrm{H} 4$ & 0.9500 & $\mathrm{C} 23-\mathrm{C} 24$ & $1.379(4)$ \\
\hline $\mathrm{C} 8-\mathrm{C} 9$ & $1.471(3)$ & $\mathrm{C} 23-\mathrm{H} 14$ & 0.9500 \\
\hline $\mathrm{C} 9-\mathrm{C} 10$ & $1.390(3)$ & $\mathrm{C} 24-\mathrm{H} 15$ & 0.9500 \\
\hline $\mathrm{C} 10-\mathrm{C} 11$ & $1.393(3)$ & & \\
\hline $\mathrm{C} 3-\mathrm{Mn} 1-\mathrm{C} 1$ & $87.58(13)$ & $\mathrm{C} 9-\mathrm{C} 10-\mathrm{H} 5$ & 120.0 \\
\hline $\mathrm{C} 3-\mathrm{Mn} 1-\mathrm{C} 2$ & $86.53(13)$ & $\mathrm{C} 11-\mathrm{C} 10-\mathrm{H} 5$ & 120.0 \\
\hline $\mathrm{C} 1-\mathrm{Mn} 1-\mathrm{C} 2$ & $90.48(13)$ & $\mathrm{C} 12-\mathrm{C} 11-\mathrm{C} 10$ & $116.6(2)$ \\
\hline $\mathrm{C} 3-\mathrm{Mn} 1-\mathrm{N} 1$ & $95.30(10)$ & $\mathrm{C} 12-\mathrm{C} 11-\mathrm{C} 19$ & $121.2(2)$ \\
\hline $\mathrm{C} 1-\mathrm{Mn} 1-\mathrm{N} 1$ & $95.53(11)$ & $\mathrm{C} 10-\mathrm{C} 11-\mathrm{C} 19$ & $122.3(2)$ \\
\hline $\mathrm{C} 2-\mathrm{Mn} 1-\mathrm{N} 1$ & $173.78(11)$ & $\mathrm{C} 13-\mathrm{C} 12-\mathrm{C} 11$ & $121.2(2)$ \\
\hline $\mathrm{C} 3-\mathrm{Mn} 1-\mathrm{N} 2$ & $172.86(11)$ & $\mathrm{C} 13-\mathrm{C} 12-\mathrm{H} 6$ & 119.4 \\
\hline $\mathrm{C} 1-\mathrm{Mn} 1-\mathrm{N} 2$ & $96.60(10)$ & $\mathrm{C} 11-\mathrm{C} 12-\mathrm{H} 6$ & 119.4 \\
\hline $\mathrm{C} 2-\mathrm{Mn} 1-\mathrm{N} 2$ & 99.18 (11) & $\mathrm{N} 2-\mathrm{C} 13-\mathrm{C} 12$ & $121.9(2)$ \\
\hline $\mathrm{N} 1-\mathrm{Mn} 1-\mathrm{N} 2$ & $78.58(8)$ & $\mathrm{N} 2-\mathrm{C} 13-\mathrm{C} 14$ & $120.3(2)$ \\
\hline $\mathrm{C} 3-\mathrm{Mn} 1-\mathrm{Br} 1$ & 89.33 (9) & $\mathrm{C} 12-\mathrm{C} 13-\mathrm{C} 14$ & $117.7(2)$ \\
\hline $\mathrm{C} 1-\mathrm{Mn} 1-\mathrm{Br} 1$ & $176.28(9)$ & $\mathrm{N} 3-\mathrm{C} 14-\mathrm{C} 15$ & $122.7(2)$ \\
\hline $\mathrm{C} 2-\mathrm{Mn} 1-\mathrm{Br} 1$ & $87.27(9)$ & $\mathrm{N} 3-\mathrm{C} 14-\mathrm{C} 13$ & $116.2(2)$ \\
\hline $\mathrm{N} 1-\mathrm{Mn} 1-\mathrm{Br} 1$ & $86.80(6)$ & $\mathrm{C} 15-\mathrm{C} 14-\mathrm{C} 13$ & $121.0(2)$ \\
\hline $\mathrm{N} 2-\mathrm{Mn} 1-\mathrm{Br} 1$ & $86.69(6)$ & $\mathrm{C} 14-\mathrm{C} 15-\mathrm{C} 16$ & 119.1 (3) \\
\hline $\mathrm{C} 4-\mathrm{N} 1-\mathrm{C} 8$ & $117.9(2)$ & $\mathrm{C} 14-\mathrm{C} 15-\mathrm{H} 7$ & 120.4 \\
\hline $\mathrm{C} 4-\mathrm{N} 1-\mathrm{Mn} 1$ & $126.07(17)$ & $\mathrm{C} 16-\mathrm{C} 15-\mathrm{H} 7$ & 120.4 \\
\hline $\mathrm{C} 8-\mathrm{N} 1-\mathrm{Mn} 1$ & $115.98(16)$ & $\mathrm{C} 17-\mathrm{C} 16-\mathrm{C} 15$ & $118.9(3)$ \\
\hline $\mathrm{C} 9-\mathrm{N} 2-\mathrm{C} 13$ & $117.3(2)$ & $\mathrm{C} 17-\mathrm{C} 16-\mathrm{H} 8$ & 120.6 \\
\hline
\end{tabular}




\begin{tabular}{|c|c|c|c|}
\hline $\mathrm{C} 9-\mathrm{N} 2-\mathrm{Mn} 1$ & $113.14(16)$ & $\mathrm{C} 15-\mathrm{C} 16-\mathrm{H} 8$ & 120.6 \\
\hline $\mathrm{C} 13-\mathrm{N} 2-\mathrm{Mn} 1$ & $128.74(17)$ & $\mathrm{C} 18-\mathrm{C} 17-\mathrm{C} 16$ & $118.4(3)$ \\
\hline $\mathrm{C} 18-\mathrm{N} 3-\mathrm{C} 14$ & $117.3(2)$ & $\mathrm{C} 18-\mathrm{C} 17-\mathrm{H} 9$ & 120.8 \\
\hline $\mathrm{O} 1-\mathrm{C} 1-\mathrm{Mn} 1$ & $174.2(2)$ & $\mathrm{C} 16-\mathrm{C} 17-\mathrm{H} 9$ & 120.8 \\
\hline $\mathrm{O} 2-\mathrm{C} 2-\mathrm{Mn} 1$ & $175.2(3)$ & $\mathrm{N} 3-\mathrm{C} 18-\mathrm{C} 17$ & $123.6(3)$ \\
\hline $\mathrm{O} 3-\mathrm{C} 3-\mathrm{Mn} 1$ & $177.3(3)$ & $\mathrm{N} 3-\mathrm{C} 18-\mathrm{H} 10$ & 118.2 \\
\hline $\mathrm{N} 1-\mathrm{C} 4-\mathrm{C} 5$ & $123.3(2)$ & $\mathrm{C} 17-\mathrm{C} 18-\mathrm{H} 10$ & 118.2 \\
\hline $\mathrm{N} 1-\mathrm{C} 4-\mathrm{H} 1$ & 118.4 & $\mathrm{C} 24-\mathrm{C} 19-\mathrm{C} 20$ & $117.6(2)$ \\
\hline $\mathrm{C} 5-\mathrm{C} 4-\mathrm{H} 1$ & 118.4 & $\mathrm{C} 24-\mathrm{C} 19-\mathrm{C} 11$ & $120.9(2)$ \\
\hline $\mathrm{C} 4-\mathrm{C} 5-\mathrm{C} 6$ & $118.8(3)$ & $\mathrm{C} 20-\mathrm{C} 19-\mathrm{C} 11$ & $121.5(2)$ \\
\hline $\mathrm{C} 4-\mathrm{C} 5-\mathrm{H} 2$ & 120.6 & $\mathrm{C} 21-\mathrm{C} 20-\mathrm{C} 19$ & $121.1(2)$ \\
\hline $\mathrm{C} 6-\mathrm{C} 5-\mathrm{H} 2$ & 120.6 & $\mathrm{C} 21-\mathrm{C} 20-\mathrm{H} 11$ & 119.5 \\
\hline $\mathrm{C} 7-\mathrm{C} 6-\mathrm{C} 5$ & $118.6(3)$ & $\mathrm{C} 19-\mathrm{C} 20-\mathrm{H} 11$ & 119.5 \\
\hline $\mathrm{C} 7-\mathrm{C} 6-\mathrm{H} 3$ & 120.7 & $\mathrm{C} 22-\mathrm{C} 21-\mathrm{C} 20$ & $119.8(3)$ \\
\hline $\mathrm{C} 5-\mathrm{C} 6-\mathrm{H} 3$ & 120.7 & $\mathrm{C} 22-\mathrm{C} 21-\mathrm{H} 12$ & 120.1 \\
\hline $\mathrm{C} 6-\mathrm{C} 7-\mathrm{C} 8$ & $119.7(2)$ & $\mathrm{C} 20-\mathrm{C} 21-\mathrm{H} 12$ & 120.1 \\
\hline $\mathrm{C} 6-\mathrm{C} 7-\mathrm{H} 4$ & 120.2 & $\mathrm{C} 23-\mathrm{C} 22-\mathrm{C} 21$ & $119.3(2)$ \\
\hline $\mathrm{C} 8-\mathrm{C} 7-\mathrm{H} 4$ & 120.2 & $\mathrm{C} 23-\mathrm{C} 22-\mathrm{H} 13$ & 120.3 \\
\hline $\mathrm{N} 1-\mathrm{C} 8-\mathrm{C} 7$ & $121.7(2)$ & $\mathrm{C} 21-\mathrm{C} 22-\mathrm{H} 13$ & 120.3 \\
\hline $\mathrm{N} 1-\mathrm{C} 8-\mathrm{C} 9$ & $114.5(2)$ & $\mathrm{C} 22-\mathrm{C} 23-\mathrm{C} 24$ & $120.9(3)$ \\
\hline $\mathrm{C} 7-\mathrm{C} 8-\mathrm{C} 9$ & $123.7(2)$ & $\mathrm{C} 22-\mathrm{C} 23-\mathrm{H} 14$ & 119.6 \\
\hline $\mathrm{N} 2-\mathrm{C} 9-\mathrm{C} 10$ & $122.9(2)$ & $\mathrm{C} 24-\mathrm{C} 23-\mathrm{H} 14$ & 119.6 \\
\hline $\mathrm{N} 2-\mathrm{C} 9-\mathrm{C} 8$ & $115.1(2)$ & $\mathrm{C} 19-\mathrm{C} 24-\mathrm{C} 23$ & $121.2(3)$ \\
\hline $\mathrm{C} 10-\mathrm{C} 9-\mathrm{C} 8$ & $122.0(2)$ & $\mathrm{C} 19-\mathrm{C} 24-\mathrm{H} 15$ & 119.4 \\
\hline $\mathrm{C} 9-\mathrm{C} 10-\mathrm{C} 11$ & $120.0(2)$ & $\mathrm{C} 23-\mathrm{C} 24-\mathrm{H} 15$ & 119.4 \\
\hline $\mathrm{C} 8-\mathrm{N} 1-\mathrm{C} 4-\mathrm{C} 5$ & $1.8(4)$ & $\mathrm{Mn} 1-\mathrm{N} 2-\mathrm{C} 13-\mathrm{C} 14$ & $20.3(3)$ \\
\hline $\mathrm{Mn} 1-\mathrm{N} 1-\mathrm{C} 4-\mathrm{C} 5$ & $178.9(2)$ & $\mathrm{C} 11-\mathrm{C} 12-\mathrm{C} 13-\mathrm{N} 2$ & $-1.1(4)$ \\
\hline $\mathrm{N} 1-\mathrm{C} 4-\mathrm{C} 5-\mathrm{C} 6$ & $-1.9(5)$ & $\mathrm{C} 11-\mathrm{C} 12-\mathrm{C} 13-\mathrm{C} 14$ & $175.4(2)$ \\
\hline $\mathrm{C} 4-\mathrm{C} 5-\mathrm{C} 6-\mathrm{C} 7$ & $0.4(4)$ & $\mathrm{C} 18-\mathrm{N} 3-\mathrm{C} 14-\mathrm{C} 15$ & $-0.3(4)$ \\
\hline $\mathrm{C} 5-\mathrm{C} 6-\mathrm{C} 7-\mathrm{C} 8$ & $1.0(4)$ & $\mathrm{C} 18-\mathrm{N} 3-\mathrm{C} 14-\mathrm{C} 13$ & $177.8(3)$ \\
\hline $\mathrm{C} 4-\mathrm{N} 1-\mathrm{C} 8-\mathrm{C} 7$ & $-0.3(4)$ & $\mathrm{N} 2-\mathrm{C} 13-\mathrm{C} 14-\mathrm{N} 3$ & $47.9(3)$ \\
\hline $\mathrm{Mn} 1-\mathrm{N} 1-\mathrm{C} 8-\mathrm{C} 7$ & $-177.7(2)$ & $\mathrm{C} 12-\mathrm{C} 13-\mathrm{C} 14-\mathrm{N} 3$ & $-128.7(3)$ \\
\hline $\mathrm{C} 4-\mathrm{N} 1-\mathrm{C} 8-\mathrm{C} 9$ & $-177.3(2)$ & $\mathrm{N} 2-\mathrm{C} 13-\mathrm{C} 14-\mathrm{C} 15$ & $-134.0(3)$ \\
\hline $\mathrm{Mn} 1-\mathrm{N} 1-\mathrm{C} 8-\mathrm{C} 9$ & $5.3(3)$ & $\mathrm{C} 12-\mathrm{C} 13-\mathrm{C} 14-\mathrm{C} 15$ & $49.5(4)$ \\
\hline $\mathrm{C} 6-\mathrm{C} 7-\mathrm{C} 8-\mathrm{N} 1$ & $-1.1(4)$ & $\mathrm{N} 3-\mathrm{C} 14-\mathrm{C} 15-\mathrm{C} 16$ & $-0.1(4)$ \\
\hline $\mathrm{C} 6-\mathrm{C} 7-\mathrm{C} 8-\mathrm{C} 9$ & $175.7(2)$ & $\mathrm{C} 13-\mathrm{C} 14-\mathrm{C} 15-\mathrm{C} 16$ & $-178.1(3)$ \\
\hline $\mathrm{C} 13-\mathrm{N} 2-\mathrm{C} 9-\mathrm{C} 10$ & $-5.6(3)$ & $\mathrm{C} 14-\mathrm{C} 15-\mathrm{C} 16-\mathrm{C} 17$ & $0.4(5)$ \\
\hline $\mathrm{Mn} 1-\mathrm{N} 2-\mathrm{C} 9-\mathrm{C} 10$ & $164.82(19)$ & $\mathrm{C} 15-\mathrm{C} 16-\mathrm{C} 17-\mathrm{C} 18$ & $-0.3(5)$ \\
\hline $\mathrm{C} 13-\mathrm{N} 2-\mathrm{C} 9-\mathrm{C} 8$ & $173.0(2)$ & $\mathrm{C} 14-\mathrm{N} 3-\mathrm{C} 18-\mathrm{C} 17$ & $0.5(5)$ \\
\hline $\mathrm{Mn} 1-\mathrm{N} 2-\mathrm{C} 9-\mathrm{C} 8$ & $-16.7(3)$ & $\mathrm{C} 16-\mathrm{C} 17-\mathrm{C} 18-\mathrm{N} 3$ & $-0.2(5)$ \\
\hline $\mathrm{N} 1-\mathrm{C} 8-\mathrm{C} 9-\mathrm{N} 2$ & $7.9(3)$ & $\mathrm{C} 12-\mathrm{C} 11-\mathrm{C} 19-\mathrm{C} 24$ & $-10.3(4)$ \\
\hline $\mathrm{C} 7-\mathrm{C} 8-\mathrm{C} 9-\mathrm{N} 2$ & $-169.1(2)$ & $\mathrm{C} 10-\mathrm{C} 11-\mathrm{C} 19-\mathrm{C} 24$ & $170.7(3)$ \\
\hline $\mathrm{N} 1-\mathrm{C} 8-\mathrm{C} 9-\mathrm{C} 10$ & $-173.6(2)$ & $\mathrm{C} 12-\mathrm{C} 11-\mathrm{C} 19-\mathrm{C} 20$ & $169.1(2)$ \\
\hline $\mathrm{C} 7-\mathrm{C} 8-\mathrm{C} 9-\mathrm{C} 10$ & $9.4(4)$ & $\mathrm{C} 10-\mathrm{C} 11-\mathrm{C} 19-\mathrm{C} 20$ & $-9.9(4)$ \\
\hline $\mathrm{N} 2-\mathrm{C} 9-\mathrm{C} 10-\mathrm{C} 11$ & $1.4(4)$ & $\mathrm{C} 24-\mathrm{C} 19-\mathrm{C} 20-\mathrm{C} 21$ & $-1.8(4)$ \\
\hline $\mathrm{C} 8-\mathrm{C} 9-\mathrm{C} 10-\mathrm{C} 11$ & $-177.0(2)$ & $\mathrm{C} 11-\mathrm{C} 19-\mathrm{C} 20-\mathrm{C} 21$ & $178.7(3)$ \\
\hline $\mathrm{C} 9-\mathrm{C} 10-\mathrm{C} 11-\mathrm{C} 12$ & $2.9(3)$ & $\mathrm{C} 19-\mathrm{C} 20-\mathrm{C} 21-\mathrm{C} 22$ & $-0.2(5)$ \\
\hline
\end{tabular}




$\begin{array}{llll}\mathrm{C} 9-\mathrm{C} 10-\mathrm{C} 11-\mathrm{C} 19 & -178.0(2) & \mathrm{C} 20-\mathrm{C} 21-\mathrm{C} 22-\mathrm{C} 23 & 2.7(5) \\ \mathrm{C} 10-\mathrm{C} 11-\mathrm{C} 12-\mathrm{C} 13 & -3.1(4) & \mathrm{C} 21-\mathrm{C} 22-\mathrm{C} 23-\mathrm{C} 24 & -3.1(5) \\ \mathrm{C} 19-\mathrm{C} 11-\mathrm{C} 12-\mathrm{C} 13 & 177.8(2) & \mathrm{C} 20-\mathrm{C} 19-\mathrm{C} 24-\mathrm{C} 23 & 1.4(5) \\ \mathrm{C} 9-\mathrm{N} 2-\mathrm{C} 13-\mathrm{C} 12 & 5.3(3) & \mathrm{C} 11-\mathrm{C} 19-\mathrm{C} 24-\mathrm{C} 23 & -179.1(3) \\ \mathrm{Mn} 1-\mathrm{N} 2-\mathrm{C} 13-\mathrm{C} 12 & -163.30(18) & \mathrm{C} 22-\mathrm{C} 23-\mathrm{C} 24-\mathrm{C} 19 & 1.0(6) \\ \mathrm{C} 9-\mathrm{N} 2-\mathrm{C} 13-\mathrm{C} 14 & -171.0(2) & & \end{array}$

Hydrogen-bond geometry $\left(\AA,{ }^{\circ}\right)$

\begin{tabular}{lllll}
\hline$D-\mathrm{H} \cdots A$ & $D-\mathrm{H}$ & $\mathrm{H} \cdots A$ & $D \cdots A$ & $D-\mathrm{H} \cdots A$ \\
\hline $\mathrm{C} 7-\mathrm{H} 4 \cdots \mathrm{Br}^{1}{ }^{\mathrm{i}}$ & 0.95 & 2.83 & $3.754(3)$ & 165 \\
$\mathrm{C} 16-\mathrm{H} 8 \cdots \mathrm{Br} 1^{1 i}$ & 0.95 & 2.88 & $3.612(4)$ & 135 \\
$\mathrm{C} 20-\mathrm{H} 11 \cdots \mathrm{Br}^{\mathrm{i}}$ & 0.95 & 2.92 & $3.844(2)$ & 163 \\
\hline
\end{tabular}

Symmetry codes: (i) $-x+1, y-1 / 2,-z+3 / 2$; (ii) $-x+1, y+1 / 2,-z+3 / 2$.

cis-Bromidodicarbonyl(4'-phenyl-2, 2': $6^{\prime}, 2^{\prime \prime}$-terpyridine- $\left.\kappa^{3} N, N^{\prime}, N^{\prime \prime}\right)$ manganese(I) (II)

Crystal data

$\left[\mathrm{MnBr}\left(\mathrm{C}_{21} \mathrm{H}_{15} \mathrm{~N}_{3}\right)(\mathrm{CO})_{2}\right]$

$M_{r}=500.23$

Monoclinic, $P 2_{1} / c$

$a=10.497(3) \AA$

$b=14.123(5) \AA$

$c=13.504(4) \AA$

$\beta=96.767(3)^{\circ}$

$V=1988.0(11) \AA^{3}$

$Z=4$

\section{Data collection}

Rigaku Saturn70

diffractometer

Detector resolution: 28.626 pixels $\mathrm{mm}^{-1}$

$\omega$ scans

Absorption correction: multi-scan (REQAB, Rigaku, 1998)

$T_{\min }=0.795, T_{\max }=0.873$

19872 measured reflections

\section{Refinement}

Refinement on $F^{2}$

$R\left[F^{2}>2 \sigma\left(F^{2}\right)\right]=0.046$

$w R\left(F^{2}\right)=0.096$

$S=1.27$

4518 reflections

289 parameters

3 restraints

Primary atom site location: structure-invariant direct methods
$F(000)=1000.00$

$D_{\mathrm{x}}=1.671 \mathrm{Mg} \mathrm{m}^{-3}$

Mo $K \alpha$ radiation, $\lambda=0.71075 \AA$

Cell parameters from 5160 reflections

$\theta=3.0-27.5^{\circ}$

$\mu=2.71 \mathrm{~mm}^{-1}$

$T=93 \mathrm{~K}$

Block, red

$0.20 \times 0.08 \times 0.05 \mathrm{~mm}$

4518 independent reflections

4016 reflections with $F^{2}>2.0 \sigma\left(F^{2}\right)$

$R_{\text {int }}=0.028$

$\theta_{\max }=27.5^{\circ}, \theta_{\min }=3.0^{\circ}$

$h=-13 \rightarrow 13$

$k=-18 \rightarrow 18$

$l=-17 \rightarrow 17$

Secondary atom site location: difference Fourier map

Hydrogen site location: inferred from neighbouring sites

$\mathrm{H}$-atom parameters constrained

$w=1 /\left[\sigma^{2}\left(F_{\mathrm{o}}^{2}\right)+(0.0039 P)^{2}+5.6867 P\right]$

where $P=\left(F_{\mathrm{o}}^{2}+2 F_{\mathrm{c}}^{2}\right) / 3$

$(\Delta / \sigma)_{\max }<0.001$

$\Delta \rho_{\max }=0.83$ e $\AA^{-3}$

$\Delta \rho_{\min }=-0.80$ e $\AA^{-3}$ 
Special details

Refinement. Refinement was performed using all reflections. The weighted R-factor (wR) and goodness of fit (S) are based on $\mathrm{F}^{2}$. R-factor (gt) are based on $\mathrm{F}$. The threshold expression of $\mathrm{F}^{2}>2.0 \operatorname{sigma}\left(\mathrm{F}^{2}\right)$ is used only for calculating $\mathrm{R}$ factor (gt).

Fractional atomic coordinates and isotropic or equivalent isotropic displacement parameters $\left(\AA^{2}\right)$

\begin{tabular}{|c|c|c|c|c|c|}
\hline & $x$ & $y$ & $z$ & $U_{\text {iso }} * / U_{\text {eq }}$ & Occ. $(<1)$ \\
\hline $\mathrm{Br} 1$ & $0.39194(6)$ & $0.11344(3)$ & $0.73710(4)$ & $0.02535(17)$ & $0.807(2)$ \\
\hline $\mathrm{O} 2$ & $-0.0653(5)$ & $0.0445(4)$ & $0.8859(4)$ & $0.0351(15)$ & $0.807(2)$ \\
\hline $\mathrm{C} 2$ & $0.0332(9)$ & $0.0641(9)$ & $0.8536(10)$ & $0.037(3)$ & 0.807 (2) \\
\hline $\mathrm{Br} 2$ & $-0.0197(5)$ & $0.0661(4)$ & $0.8657(4)$ & $0.0403(14)$ & $0.193(2)$ \\
\hline $\mathrm{O} 3$ & $0.4377(18)$ & $0.1006(12)$ & $0.7111(13)$ & $0.033(4)^{*}$ & $0.193(2)$ \\
\hline $\mathrm{C} 3$ & 0.339 & $0.092(3)$ & $0.746(3)$ & $0.076(12)^{*}$ & 0.193 \\
\hline Mn1 & $0.18158(5)$ & $0.08786(4)$ & $0.80533(4)$ & $0.02451(14)$ & \\
\hline $\mathrm{O} 1$ & $0.1797(3)$ & $-0.10749(19)$ & $0.7376(2)$ & $0.0359(6)$ & \\
\hline N1 & $0.2796(3)$ & $0.0760(2)$ & $0.9421(2)$ & $0.0224(6)$ & \\
\hline $\mathrm{N} 2$ & $0.2005(3)$ & $0.22091(19)$ & $0.8440(2)$ & $0.0202(6)$ & \\
\hline N3 & $0.0937(3)$ & $0.1521(2)$ & $0.6822(2)$ & $0.0219(6)$ & \\
\hline $\mathrm{C} 1$ & $0.1807(4)$ & -0.0334 & $0.7637(3)$ & $0.0310(8)$ & \\
\hline $\mathrm{C} 4$ & $0.3125(3)$ & $-0.0050(3)$ & $0.9917(3)$ & $0.0275(8)$ & \\
\hline H1 & 0.288826 & -0.063547 & 0.960043 & $0.033^{*}$ & \\
\hline $\mathrm{C} 5$ & $0.3784(4)$ & $-0.0070(3)$ & $1.0856(3)$ & $0.0298(8)$ & \\
\hline $\mathrm{H} 2$ & 0.400366 & -0.065698 & 1.117441 & $0.036^{*}$ & \\
\hline $\mathrm{C} 6$ & $0.4125(4)$ & $0.0773(3)$ & $1.1335(3)$ & $0.0301(8)$ & \\
\hline $\mathrm{H} 3$ & 0.458098 & 0.077281 & 1.198549 & $0.036^{*}$ & \\
\hline $\mathrm{C} 7$ & $0.3789(3)$ & $0.1623(3)$ & $1.0847(3)$ & $0.0257(7)$ & \\
\hline $\mathrm{H} 4$ & 0.400583 & 0.221221 & 1.116233 & $0.031^{*}$ & \\
\hline $\mathrm{C} 8$ & $0.3132(3)$ & $0.1592(2)$ & $0.9894(3)$ & $0.0217(7)$ & \\
\hline $\mathrm{C} 9$ & $0.2711(3)$ & $0.2443(2)$ & $0.9303(3)$ & $0.0212(7)$ & \\
\hline $\mathrm{C} 10$ & $0.3006(3)$ & $0.3376(2)$ & $0.9548(3)$ & $0.0227(7)$ & \\
\hline H5 & 0.351261 & 0.352547 & 1.015754 & $0.027^{*}$ & \\
\hline $\mathrm{C} 11$ & $0.2548(3)$ & $0.4096(2)$ & $0.8886(3)$ & $0.0223(7)$ & \\
\hline $\mathrm{C} 12$ & 0.1818 & $0.3838(2)$ & $0.7994(3)$ & $0.0225(7)$ & \\
\hline H6 & 0.149023 & 0.431227 & 0.753384 & $0.027^{*}$ & \\
\hline $\mathrm{C} 13$ & $0.1572(3)$ & $0.2887(2)$ & $0.7779(3)$ & $0.0208(7)$ & \\
\hline $\mathrm{C} 14$ & 0.0918 (3) & $0.2483(2)$ & $0.6854(3)$ & $0.0210(7)$ & \\
\hline $\mathrm{C} 15$ & 0.0335 & $0.3016(3)$ & $0.6061(3)$ & $0.0251(7)$ & \\
\hline H7 & 0.033795 & 0.368774 & 0.609582 & $0.030^{*}$ & \\
\hline $\mathrm{C} 16$ & $-0.0251(3)$ & 0.2560 & $0.5220(3)$ & $0.0274(8)$ & \\
\hline $\mathrm{H} 8$ & -0.066553 & 0.291366 & 0.467603 & $0.033^{*}$ & \\
\hline $\mathrm{C} 17$ & $-0.0223(3)$ & $0.1587(3)$ & $0.5187(3)$ & $0.0277(8)$ & \\
\hline H9 & -0.060962 & 0.125743 & 0.461531 & $0.033^{*}$ & \\
\hline $\mathrm{C} 18$ & 0.0376 & $0.1095(3)$ & $0.5994(3)$ & $0.0262(7)$ & \\
\hline H10 & 0.039210 & 0.042307 & 0.596314 & $0.031^{*}$ & \\
\hline $\mathrm{C} 19$ & 0.2863 & $0.5109(2)$ & $0.9128(3)$ & $0.0247(7)$ & \\
\hline $\mathrm{C} 20$ & $0.3268(4)$ & $0.5390(3)$ & $1.0100(3)$ & $0.0316(8)$ & \\
\hline H11 & 0.333688 & 0.493598 & 1.062254 & $0.038^{*}$ & \\
\hline
\end{tabular}




$\begin{array}{lllll}\mathrm{C} 21 & 0.3573(4) & 0.6332(3) & 1.0315(3) & 0.0385(10) \\ \mathrm{H} 12 & 0.385755 & 0.651422 & 1.098118 & 0.046^{*} \\ \mathrm{C} 22 & 0.3466(4) & 0.7000(3) & 0.9572(4) & 0.0379(10) \\ \mathrm{H} 13 & 0.367454 & 0.764157 & 0.972651 & 0.045^{*} \\ \mathrm{C} 23 & 0.3056(4) & 0.6739(3) & 0.8598(3) & 0.0376(10) \\ \mathrm{H} 14 & 0.297827 & 0.720240 & 0.808386 & 0.045^{*} \\ \mathrm{C} 24 & 0.2757(4) & 0.5797(3) & 0.8373(3) & 0.0299(8) \\ \mathrm{H} 15 & 0.247979 & 0.561819 & 0.770438 & 0.036^{*}\end{array}$

Atomic displacement parameters $\left(\AA^{2}\right)$

\begin{tabular}{|c|c|c|c|c|c|c|}
\hline & $U^{11}$ & $U^{22}$ & $U^{33}$ & $U^{12}$ & $U^{13}$ & $U^{23}$ \\
\hline Br1 & $0.0222(3)$ & $0.0293(3)$ & $0.0246(3)$ & $0.0054(2)$ & $0.0032(2)$ & $0.00736(19)$ \\
\hline $\mathrm{O} 2$ & $0.038(3)$ & $0.028(3)$ & $0.041(3)$ & $-0.0170(18)$ & $0.012(2)$ & $0.000(2)$ \\
\hline $\mathrm{C} 2$ & $0.055(7)$ & $0.023(3)$ & $0.031(4)$ & $0.009(5)$ & $-0.006(5)$ & $-0.006(3)$ \\
\hline $\mathrm{Br} 2$ & $0.060(4)$ & $0.0228(17)$ & $0.035(2)$ & $0.001(3)$ & $-0.005(3)$ & $-0.0077(14)$ \\
\hline Mn1 & $0.0276(3)$ & $0.0170(3)$ & $0.0275(3)$ & $0.0001(2)$ & $-0.0027(2)$ & $0.0030(2)$ \\
\hline $\mathrm{O} 1$ & $0.0504(18)$ & $0.0244(14)$ & $0.0342(15)$ & $0.0084(12)$ & $0.0102(13)$ & $0.0043(12)$ \\
\hline N1 & $0.0202(14)$ & $0.0197(14)$ & $0.0276(15)$ & $0.0010(11)$ & $0.0042(12)$ & $0.0048(12)$ \\
\hline N2 & $0.0176(13)$ & $0.0179(13)$ & $0.0250(15)$ & $0.0001(10)$ & $0.0017(11)$ & $0.0029(11)$ \\
\hline N3 & $0.0191(14)$ & $0.0215(14)$ & $0.0249(15)$ & $0.0006(11)$ & $0.0016(12)$ & $0.0015(12)$ \\
\hline $\mathrm{C} 1$ & $0.0266(19)$ & $0.037(2)$ & $0.029(2)$ & $-0.0021(16)$ & $0.0024(15)$ & $0.0106(17)$ \\
\hline $\mathrm{C} 4$ & $0.0248(18)$ & $0.0231(17)$ & $0.035(2)$ & $0.0036(14)$ & $0.0064(15)$ & $0.0077(15)$ \\
\hline $\mathrm{C} 5$ & $0.0276(19)$ & $0.0283(19)$ & $0.034(2)$ & $0.0070(15)$ & $0.0064(16)$ & $0.0115(16)$ \\
\hline C6 & $0.0286(19)$ & $0.039(2)$ & $0.0235(18)$ & $0.0076(16)$ & $0.0040(15)$ & $0.0082(16)$ \\
\hline $\mathrm{C} 7$ & $0.0239(17)$ & $0.0283(18)$ & $0.0252(18)$ & $0.0022(14)$ & $0.0041(14)$ & $0.0017(14)$ \\
\hline $\mathrm{C} 8$ & $0.0182(16)$ & $0.0240(17)$ & $0.0236(17)$ & $0.0012(13)$ & $0.0059(13)$ & $0.0031(14)$ \\
\hline C9 & $0.0177(16)$ & $0.0214(16)$ & $0.0249(17)$ & $0.0006(13)$ & $0.0042(13)$ & $0.0030(13)$ \\
\hline C10 & $0.0192(16)$ & $0.0247(17)$ & $0.0244(18)$ & $0.0003(13)$ & $0.0028(13)$ & $-0.0012(14)$ \\
\hline C11 & $0.0180(16)$ & $0.0202(16)$ & $0.0297(18)$ & $0.0012(12)$ & $0.0064(14)$ & $0.0008(14)$ \\
\hline $\mathrm{C} 12$ & $0.0208(16)$ & $0.0196(16)$ & $0.0270(18)$ & $0.0014(13)$ & $0.0028(13)$ & $0.0027(14)$ \\
\hline $\mathrm{C} 13$ & $0.0172(15)$ & $0.0224(17)$ & $0.0232(17)$ & $0.0012(12)$ & $0.0036(13)$ & $0.0041(13)$ \\
\hline C14 & $0.0173(15)$ & $0.0209(16)$ & $0.0251(17)$ & $0.0000(12)$ & $0.0037(13)$ & $0.0018(13)$ \\
\hline $\mathrm{C} 15$ & $0.0221(17)$ & $0.0243(17)$ & $0.0289(19)$ & $0.0016(14)$ & $0.0023(14)$ & $0.0057(14)$ \\
\hline $\mathrm{C} 16$ & $0.0225(17)$ & $0.0333(19)$ & $0.0261(19)$ & $0.0011(15)$ & $0.0016(14)$ & $0.0059(15)$ \\
\hline $\mathrm{C} 17$ & $0.0227(18)$ & $0.036(2)$ & $0.0241(18)$ & $-0.0009(15)$ & $0.0013(14)$ & $-0.0016(15)$ \\
\hline $\mathrm{C} 18$ & $0.0224(17)$ & $0.0247(17)$ & $0.0311(19)$ & $-0.0016(14)$ & $0.0020(14)$ & $-0.0005(15)$ \\
\hline C19 & $0.0181(16)$ & $0.0208(17)$ & $0.036(2)$ & $-0.0005(13)$ & $0.0065(14)$ & $-0.0015(14)$ \\
\hline $\mathrm{C} 20$ & $0.0290(19)$ & $0.0240(18)$ & $0.042(2)$ & $0.0000(15)$ & $0.0027(17)$ & $-0.0041(16)$ \\
\hline $\mathrm{C} 21$ & $0.031(2)$ & $0.031(2)$ & $0.053(3)$ & $-0.0024(16)$ & $0.0019(19)$ & $-0.0145(19)$ \\
\hline C22 & $0.030(2)$ & $0.0206(18)$ & $0.065(3)$ & $-0.0047(15)$ & $0.014(2)$ & $-0.0097(19)$ \\
\hline C23 & $0.038(2)$ & $0.0211(18)$ & $0.057(3)$ & $-0.0022(16)$ & $0.018(2)$ & $0.0041(18)$ \\
\hline C24 & $0.0288(19)$ & $0.0221(17)$ & $0.040(2)$ & $0.0013(14)$ & $0.0081(16)$ & $0.0006(16)$ \\
\hline
\end{tabular}

Geometric parameters $\left(\AA,{ }^{o}\right)$

\begin{tabular}{llll}
\hline $\mathrm{Br} 1-\mathrm{O} 3$ & $0.65(2)$ & $\mathrm{C} 9-\mathrm{C} 10$ & $1.384(5)$ \\
$\mathrm{Br} 1-\mathrm{C} 3$ & $0.66(3)$ & $\mathrm{C} 10-\mathrm{C} 11$ & $1.401(5)$
\end{tabular}




\begin{tabular}{|c|c|c|c|}
\hline $\mathrm{Br} 1-\mathrm{Mn} 1$ & $2.5170(11)$ & $\mathrm{C} 10-\mathrm{H} 5$ & 0.9500 \\
\hline $\mathrm{O} 2-\mathrm{Br} 2$ & $0.654(5)$ & $\mathrm{C} 11-\mathrm{C} 12$ & $1.398(5)$ \\
\hline $\mathrm{O} 2-\mathrm{C} 2$ & $1.201(11)$ & $\mathrm{C} 11-\mathrm{C} 19$ & $1.496(5)$ \\
\hline $\mathrm{C} 2-\mathrm{Br} 2$ & $0.597(8)$ & $\mathrm{C} 12-\mathrm{C} 13$ & $1.392(5)$ \\
\hline $\mathrm{C} 2-\mathrm{Mn} 1$ & $1.790(9)$ & $\mathrm{C} 12-\mathrm{H} 6$ & 0.9500 \\
\hline $\mathrm{O} 3-\mathrm{C} 3$ & $1.194(18)$ & $\mathrm{C} 13-\mathrm{C} 14$ & $1.468(5)$ \\
\hline $\mathrm{Mn} 1-\mathrm{C} 1$ & $1.803(4)$ & $\mathrm{C} 14-\mathrm{C} 15$ & $1.390(5)$ \\
\hline $\mathrm{Mn} 1-\mathrm{N} 2$ & $1.954(3)$ & $\mathrm{C} 15-\mathrm{C} 16$ & $1.384(5)$ \\
\hline $\mathrm{Mn} 1-\mathrm{N} 1$ & $2.012(3)$ & $\mathrm{C} 15-\mathrm{H} 7$ & 0.9500 \\
\hline $\mathrm{Mn} 1-\mathrm{N} 3$ & $2.019(3)$ & $\mathrm{C} 16-\mathrm{C} 17$ & $1.376(5)$ \\
\hline $\mathrm{O} 1-\mathrm{C} 1$ & $1.103(5)$ & $\mathrm{C} 16-\mathrm{H} 8$ & 0.9500 \\
\hline $\mathrm{N} 1-\mathrm{C} 4$ & $1.350(4)$ & $\mathrm{C} 17-\mathrm{C} 18$ & $1.380(5)$ \\
\hline $\mathrm{N} 1-\mathrm{C} 8$ & $1.364(4)$ & $\mathrm{C} 17-\mathrm{H} 9$ & 0.9500 \\
\hline $\mathrm{N} 2-\mathrm{C} 9$ & $1.346(4)$ & $\mathrm{C} 18-\mathrm{H} 10$ & 0.9500 \\
\hline $\mathrm{N} 2-\mathrm{C} 13$ & $1.351(4)$ & $\mathrm{C} 19-\mathrm{C} 20$ & $1.390(5)$ \\
\hline $\mathrm{N} 3-\mathrm{C} 18$ & $1.343(4)$ & $\mathrm{C} 19-\mathrm{C} 24$ & $1.402(5)$ \\
\hline $\mathrm{N} 3-\mathrm{C} 14$ & $1.360(4)$ & $\mathrm{C} 20-\mathrm{C} 21$ & $1.390(5)$ \\
\hline $\mathrm{C} 4-\mathrm{C} 5$ & $1.371(5)$ & $\mathrm{C} 20-\mathrm{H} 11$ & 0.9500 \\
\hline $\mathrm{C} 4-\mathrm{H} 1$ & 0.9500 & $\mathrm{C} 21-\mathrm{C} 22$ & $1.371(6)$ \\
\hline $\mathrm{C} 5-\mathrm{C} 6$ & $1.381(6)$ & $\mathrm{C} 21-\mathrm{H} 12$ & 0.9500 \\
\hline $\mathrm{C} 5-\mathrm{H} 2$ & 0.9500 & $\mathrm{C} 22-\mathrm{C} 23$ & $1.385(6)$ \\
\hline $\mathrm{C} 6-\mathrm{C} 7$ & $1.395(5)$ & $\mathrm{C} 22-\mathrm{H} 13$ & 0.9500 \\
\hline $\mathrm{C} 6-\mathrm{H} 3$ & 0.9500 & $\mathrm{C} 23-\mathrm{C} 24$ & $1.393(5)$ \\
\hline $\mathrm{C} 7-\mathrm{C} 8$ & $1.387(5)$ & $\mathrm{C} 23-\mathrm{H} 14$ & 0.9500 \\
\hline $\mathrm{C} 7-\mathrm{H} 4$ & 0.9500 & $\mathrm{C} 24-\mathrm{H} 15$ & 0.9500 \\
\hline $\mathrm{C} 8-\mathrm{C} 9$ & $1.481(5)$ & & \\
\hline $\mathrm{O} 3-\mathrm{Br} 1-\mathrm{C} 3$ & $131(4)$ & $\mathrm{N} 2-\mathrm{C} 9-\mathrm{C} 8$ & $111.5(3)$ \\
\hline $\mathrm{Br} 2-\mathrm{O} 2-\mathrm{C} 2$ & $15.7(10)$ & $\mathrm{C} 10-\mathrm{C} 9-\mathrm{C} 8$ & $126.8(3)$ \\
\hline $\mathrm{Br} 2-\mathrm{C} 2-\mathrm{O} 2$ & $17.2(12)$ & $\mathrm{C} 9-\mathrm{C} 10-\mathrm{C} 11$ & $119.2(3)$ \\
\hline $\mathrm{O} 2-\mathrm{C} 2-\mathrm{Mn} 1$ & $177.5(10)$ & $\mathrm{C} 9-\mathrm{C} 10-\mathrm{H} 5$ & 120.4 \\
\hline $\mathrm{C} 2-\mathrm{Br} 2-\mathrm{O} 2$ & $147(2)$ & $\mathrm{C} 11-\mathrm{C} 10-\mathrm{H} 5$ & 120.4 \\
\hline $\mathrm{Br} 1-\mathrm{O} 3-\mathrm{C} 3$ & $24(2)$ & $\mathrm{C} 12-\mathrm{C} 11-\mathrm{C} 10$ & $118.2(3)$ \\
\hline $\mathrm{Br} 1-\mathrm{C} 3-\mathrm{O} 3$ & 24.3 (19) & $\mathrm{C} 12-\mathrm{C} 11-\mathrm{C} 19$ & $121.5(3)$ \\
\hline $\mathrm{C} 2-\mathrm{Mn} 1-\mathrm{C} 1$ & $87.9(4)$ & $\mathrm{C} 10-\mathrm{C} 11-\mathrm{C} 19$ & $120.3(3)$ \\
\hline $\mathrm{C} 2-\mathrm{Mn} 1-\mathrm{N} 2$ & $98.6(4)$ & $\mathrm{C} 13-\mathrm{C} 12-\mathrm{C} 11$ & $120.0(3)$ \\
\hline $\mathrm{C} 1-\mathrm{Mn} 1-\mathrm{N} 2$ & $173.57(15)$ & $\mathrm{C} 13-\mathrm{C} 12-\mathrm{H} 6$ & 120.0 \\
\hline $\mathrm{C} 2-\mathrm{Mn} 1-\mathrm{N} 1$ & $91.3(4)$ & $\mathrm{C} 11-\mathrm{C} 12-\mathrm{H} 6$ & 120.0 \\
\hline $\mathrm{C} 1-\mathrm{Mn} 1-\mathrm{N} 1$ & $100.98(14)$ & $\mathrm{N} 2-\mathrm{C} 13-\mathrm{C} 12$ & $120.4(3)$ \\
\hline $\mathrm{N} 2-\mathrm{Mn} 1-\mathrm{N} 1$ & $79.05(12)$ & $\mathrm{N} 2-\mathrm{C} 13-\mathrm{C} 14$ & $112.0(3)$ \\
\hline $\mathrm{C} 2-\mathrm{Mn} 1-\mathrm{N} 3$ & $93.0(4)$ & $\mathrm{C} 12-\mathrm{C} 13-\mathrm{C} 14$ & $127.5(3)$ \\
\hline $\mathrm{C} 1-\mathrm{Mn} 1-\mathrm{N} 3$ & $100.66(15)$ & $\mathrm{N} 3-\mathrm{C} 14-\mathrm{C} 15$ & $121.5(3)$ \\
\hline $\mathrm{N} 2-\mathrm{Mn} 1-\mathrm{N} 3$ & $79.05(12)$ & $\mathrm{N} 3-\mathrm{C} 14-\mathrm{C} 13$ & $114.1(3)$ \\
\hline $\mathrm{N} 1-\mathrm{Mn} 1-\mathrm{N} 3$ & $158.08(12)$ & $\mathrm{C} 15-\mathrm{C} 14-\mathrm{C} 13$ & $124.4(3)$ \\
\hline $\mathrm{C} 2-\mathrm{Mn} 1-\mathrm{Br} 1$ & $177.4(4)$ & $\mathrm{C} 16-\mathrm{C} 15-\mathrm{C} 14$ & $119.6(3)$ \\
\hline $\mathrm{C} 1-\mathrm{Mn} 1-\mathrm{Br} 1$ & $89.68(12)$ & $\mathrm{C} 16-\mathrm{C} 15-\mathrm{H} 7$ & 120.2 \\
\hline $\mathrm{N} 2-\mathrm{Mn} 1-\mathrm{Br} 1$ & $83.89(8)$ & $\mathrm{C} 14-\mathrm{C} 15-\mathrm{H} 7$ & 120.2 \\
\hline $\mathrm{N} 1-\mathrm{Mn} 1-\mathrm{Br} 1$ & $88.42(8)$ & $\mathrm{C} 17-\mathrm{C} 16-\mathrm{C} 15$ & $118.8(3)$ \\
\hline
\end{tabular}




\begin{tabular}{|c|c|c|c|}
\hline $\mathrm{N} 3-\mathrm{Mn} 1-\mathrm{Br} 1$ & $88.26(8)$ & $\mathrm{C} 17-\mathrm{C} 16-\mathrm{H} 8$ & 120.6 \\
\hline $\mathrm{C} 4-\mathrm{N} 1-\mathrm{C} 8$ & $117.4(3)$ & $\mathrm{C} 15-\mathrm{C} 16-\mathrm{H} 8$ & 120.6 \\
\hline $\mathrm{C} 4-\mathrm{N} 1-\mathrm{Mn} 1$ & $126.8(3)$ & $\mathrm{C} 16-\mathrm{C} 17-\mathrm{C} 18$ & $119.1(3)$ \\
\hline $\mathrm{C} 8-\mathrm{N} 1-\mathrm{Mn} 1$ & $115.8(2)$ & $\mathrm{C} 16-\mathrm{C} 17-\mathrm{H} 9$ & 120.4 \\
\hline $\mathrm{C} 9-\mathrm{N} 2-\mathrm{C} 13$ & $120.4(3)$ & $\mathrm{C} 18-\mathrm{C} 17-\mathrm{H} 9$ & 120.4 \\
\hline $\mathrm{C} 9-\mathrm{N} 2-\mathrm{Mn} 1$ & $119.7(2)$ & $\mathrm{N} 3-\mathrm{C} 18-\mathrm{C} 17$ & $123.1(3)$ \\
\hline $\mathrm{C} 13-\mathrm{N} 2-\mathrm{Mn} 1$ & $119.3(2)$ & $\mathrm{N} 3-\mathrm{C} 18-\mathrm{H} 10$ & 118.4 \\
\hline $\mathrm{C} 18-\mathrm{N} 3-\mathrm{C} 14$ & $117.9(3)$ & $\mathrm{C} 17-\mathrm{C} 18-\mathrm{H} 10$ & 118.4 \\
\hline $\mathrm{C} 18-\mathrm{N} 3-\mathrm{Mn} 1$ & $126.7(2)$ & $\mathrm{C} 20-\mathrm{C} 19-\mathrm{C} 24$ & 118.5 \\
\hline $\mathrm{C} 14-\mathrm{N} 3-\mathrm{Mn} 1$ & $115.5(2)$ & $\mathrm{C} 20-\mathrm{C} 19-\mathrm{C} 11$ & $121.0(3)$ \\
\hline $\mathrm{O} 1-\mathrm{C} 1-\mathrm{Mn} 1$ & $179.5(4)$ & $\mathrm{C} 24-\mathrm{C} 19-\mathrm{C} 11$ & $120.5(3)$ \\
\hline $\mathrm{N} 1-\mathrm{C} 4-\mathrm{C} 5$ & $123.2(4)$ & $\mathrm{C} 21-\mathrm{C} 20-\mathrm{C} 19$ & $120.5(4)$ \\
\hline $\mathrm{N} 1-\mathrm{C} 4-\mathrm{H} 1$ & 118.4 & $\mathrm{C} 21-\mathrm{C} 20-\mathrm{H} 11$ & 119.8 \\
\hline $\mathrm{C} 5-\mathrm{C} 4-\mathrm{H} 1$ & 118.4 & $\mathrm{C} 19-\mathrm{C} 20-\mathrm{H} 11$ & 119.8 \\
\hline $\mathrm{C} 4-\mathrm{C} 5-\mathrm{C} 6$ & $119.4(3)$ & $\mathrm{C} 22-\mathrm{C} 21-\mathrm{C} 20$ & $120.6(4)$ \\
\hline $\mathrm{C} 4-\mathrm{C} 5-\mathrm{H} 2$ & 120.3 & $\mathrm{C} 22-\mathrm{C} 21-\mathrm{H} 12$ & 119.7 \\
\hline $\mathrm{C} 6-\mathrm{C} 5-\mathrm{H} 2$ & 120.3 & $\mathrm{C} 20-\mathrm{C} 21-\mathrm{H} 12$ & 119.7 \\
\hline $\mathrm{C} 5-\mathrm{C} 6-\mathrm{C} 7$ & $118.9(3)$ & $\mathrm{C} 21-\mathrm{C} 22-\mathrm{C} 23$ & $120.1(4)$ \\
\hline $\mathrm{C} 5-\mathrm{C} 6-\mathrm{H} 3$ & 120.5 & $\mathrm{C} 21-\mathrm{C} 22-\mathrm{H} 13$ & 120.0 \\
\hline $\mathrm{C} 7-\mathrm{C} 6-\mathrm{H} 3$ & 120.5 & $\mathrm{C} 23-\mathrm{C} 22-\mathrm{H} 13$ & 120.0 \\
\hline $\mathrm{C} 8-\mathrm{C} 7-\mathrm{C} 6$ & $118.8(3)$ & $\mathrm{C} 22-\mathrm{C} 23-\mathrm{C} 24$ & $119.8(4)$ \\
\hline $\mathrm{C} 8-\mathrm{C} 7-\mathrm{H} 4$ & 120.6 & $\mathrm{C} 22-\mathrm{C} 23-\mathrm{H} 14$ & 120.1 \\
\hline $\mathrm{C} 6-\mathrm{C} 7-\mathrm{H} 4$ & 120.6 & $\mathrm{C} 24-\mathrm{C} 23-\mathrm{H} 14$ & 120.1 \\
\hline $\mathrm{N} 1-\mathrm{C} 8-\mathrm{C} 7$ & $122.3(3)$ & $\mathrm{C} 23-\mathrm{C} 24-\mathrm{C} 19$ & $120.5(4)$ \\
\hline $\mathrm{N} 1-\mathrm{C} 8-\mathrm{C} 9$ & $113.7(3)$ & $\mathrm{C} 23-\mathrm{C} 24-\mathrm{H} 15$ & 119.8 \\
\hline $\mathrm{C} 7-\mathrm{C} 8-\mathrm{C} 9$ & $124.0(3)$ & $\mathrm{C} 19-\mathrm{C} 24-\mathrm{H} 15$ & 119.8 \\
\hline $\mathrm{N} 2-\mathrm{C} 9-\mathrm{C} 10$ & $121.7(3)$ & & \\
\hline $\mathrm{C} 8-\mathrm{N} 1-\mathrm{C} 4-\mathrm{C} 5$ & $-0.7(5)$ & $\mathrm{C} 11-\mathrm{C} 12-\mathrm{C} 13-\mathrm{N} 2$ & $1.8(5)$ \\
\hline $\mathrm{Mn} 1-\mathrm{N} 1-\mathrm{C} 4-\mathrm{C} 5$ & $-179.2(3)$ & $\mathrm{C} 11-\mathrm{C} 12-\mathrm{C} 13-\mathrm{C} 14$ & $-174.8(3)$ \\
\hline $\mathrm{N} 1-\mathrm{C} 4-\mathrm{C} 5-\mathrm{C} 6$ & $0.7(6)$ & $\mathrm{C} 18-\mathrm{N} 3-\mathrm{C} 14-\mathrm{C} 15$ & $0.3(5)$ \\
\hline $\mathrm{C} 4-\mathrm{C} 5-\mathrm{C} 6-\mathrm{C} 7$ & $0.0(5)$ & $\mathrm{Mn} 1-\mathrm{N} 3-\mathrm{C} 14-\mathrm{C} 15$ & $-179.4(3)$ \\
\hline $\mathrm{C} 5-\mathrm{C} 6-\mathrm{C} 7-\mathrm{C} 8$ & $-0.5(5)$ & $\mathrm{C} 18-\mathrm{N} 3-\mathrm{C} 14-\mathrm{C} 13$ & $-179.0(3)$ \\
\hline $\mathrm{C} 4-\mathrm{N} 1-\mathrm{C} 8-\mathrm{C} 7$ & $0.1(5)$ & $\mathrm{Mn} 1-\mathrm{N} 3-\mathrm{C} 14-\mathrm{C} 13$ & $1.3(4)$ \\
\hline $\mathrm{Mn} 1-\mathrm{N} 1-\mathrm{C} 8-\mathrm{C} 7$ & $178.8(3)$ & $\mathrm{N} 2-\mathrm{C} 13-\mathrm{C} 14-\mathrm{N} 3$ & $-3.4(4)$ \\
\hline $\mathrm{C} 4-\mathrm{N} 1-\mathrm{C} 8-\mathrm{C} 9$ & $-178.9(3)$ & $\mathrm{C} 12-\mathrm{C} 13-\mathrm{C} 14-\mathrm{N} 3$ & $173.5(3)$ \\
\hline $\mathrm{Mn} 1-\mathrm{N} 1-\mathrm{C} 8-\mathrm{C} 9$ & $-0.2(4)$ & $\mathrm{N} 2-\mathrm{C} 13-\mathrm{C} 14-\mathrm{C} 15$ & $177.4(3)$ \\
\hline $\mathrm{C} 6-\mathrm{C} 7-\mathrm{C} 8-\mathrm{N} 1$ & $0.5(5)$ & $\mathrm{C} 12-\mathrm{C} 13-\mathrm{C} 14-\mathrm{C} 15$ & $-5.7(6)$ \\
\hline $\mathrm{C} 6-\mathrm{C} 7-\mathrm{C} 8-\mathrm{C} 9$ & $179.4(3)$ & $\mathrm{N} 3-\mathrm{C} 14-\mathrm{C} 15-\mathrm{C} 16$ & $0.5(5)$ \\
\hline $\mathrm{C} 13-\mathrm{N} 2-\mathrm{C} 9-\mathrm{C} 10$ & $0.7(5)$ & $\mathrm{C} 13-\mathrm{C} 14-\mathrm{C} 15-\mathrm{C} 16$ & $179.7(3)$ \\
\hline $\mathrm{Mn} 1-\mathrm{N} 2-\mathrm{C} 9-\mathrm{C} 10$ & $171.8(2)$ & $\mathrm{C} 14-\mathrm{C} 15-\mathrm{C} 16-\mathrm{C} 17$ & $-1.0(5)$ \\
\hline $\mathrm{C} 13-\mathrm{N} 2-\mathrm{C} 9-\mathrm{C} 8$ & $-177.5(3)$ & $\mathrm{C} 15-\mathrm{C} 16-\mathrm{C} 17-\mathrm{C} 18$ & $0.7(5)$ \\
\hline $\mathrm{Mn} 1-\mathrm{N} 2-\mathrm{C} 9-\mathrm{C} 8$ & $-6.4(4)$ & $\mathrm{C} 14-\mathrm{N} 3-\mathrm{C} 18-\mathrm{C} 17$ & $-0.6(5)$ \\
\hline $\mathrm{N} 1-\mathrm{C} 8-\mathrm{C} 9-\mathrm{N} 2$ & $4.1(4)$ & $\mathrm{Mn} 1-\mathrm{N} 3-\mathrm{C} 18-\mathrm{C} 17$ & $179.1(3)$ \\
\hline $\mathrm{C} 7-\mathrm{C} 8-\mathrm{C} 9-\mathrm{N} 2$ & $-174.9(3)$ & $\mathrm{C} 16-\mathrm{C} 17-\mathrm{C} 18-\mathrm{N} 3$ & $0.1(6)$ \\
\hline $\mathrm{N} 1-\mathrm{C} 8-\mathrm{C} 9-\mathrm{C} 10$ & $-174.0(3)$ & $\mathrm{C} 12-\mathrm{C} 11-\mathrm{C} 19-\mathrm{C} 20$ & $162.1(3)$ \\
\hline $\mathrm{C} 7-\mathrm{C} 8-\mathrm{C} 9-\mathrm{C} 10$ & $7.0(5)$ & $\mathrm{C} 10-\mathrm{C} 11-\mathrm{C} 19-\mathrm{C} 20$ & $-19.3(5)$ \\
\hline $\mathrm{N} 2-\mathrm{C} 9-\mathrm{C} 10-\mathrm{C} 11$ & $0.6(5)$ & $\mathrm{C} 12-\mathrm{C} 11-\mathrm{C} 19-\mathrm{C} 24$ & $-18.0(5)$ \\
\hline
\end{tabular}




$\begin{array}{llll}\mathrm{C} 8-\mathrm{C} 9-\mathrm{C} 10-\mathrm{C} 11 & 178.5(3) & \mathrm{C} 10-\mathrm{C} 11-\mathrm{C} 19-\mathrm{C} 24 & 160.6(3) \\ \mathrm{C} 9-\mathrm{C} 10-\mathrm{C} 11-\mathrm{C} 12 & -0.7(5) & \mathrm{C} 24-\mathrm{C} 19-\mathrm{C} 20-\mathrm{C} 21 & -0.7(6) \\ \mathrm{C} 9-\mathrm{C} 10-\mathrm{C} 11-\mathrm{C} 19 & -179.4(3) & \mathrm{C} 11-\mathrm{C} 19-\mathrm{C} 20-\mathrm{C} 21 & 179.2(3) \\ \mathrm{C} 10-\mathrm{C} 11-\mathrm{C} 12-\mathrm{C} 13 & -0.5(5) & \mathrm{C} 19-\mathrm{C} 20-\mathrm{C} 21-\mathrm{C} 22 & 0.7(6) \\ \mathrm{C} 19-\mathrm{C} 11-\mathrm{C} 12-\mathrm{C} 13 & 178.2(3) & \mathrm{C} 20-\mathrm{C} 21-\mathrm{C} 22-\mathrm{C} 23 & -0.2(6) \\ \mathrm{C} 9-\mathrm{N} 2-\mathrm{C} 13-\mathrm{C} 12 & -1.9(5) & \mathrm{C} 21-\mathrm{C} 22-\mathrm{C} 23-\mathrm{C} 24 & -0.3(6) \\ \mathrm{Mn} 1-\mathrm{N} 2-\mathrm{C} 13-\mathrm{C} 12 & -173.0(2) & \mathrm{C} 22-\mathrm{C} 23-\mathrm{C} 24-\mathrm{C} 19 & 0.3(6) \\ \mathrm{C} 9-\mathrm{N} 2-\mathrm{C} 13-\mathrm{C} 14 & 175.2(3) & \mathrm{C} 20-\mathrm{C} 19-\mathrm{C} 24-\mathrm{C} 23 & 0.2(5) \\ \mathrm{Mn} 1-\mathrm{N} 2-\mathrm{C} 13-\mathrm{C} 14 & 4.1(4) & \mathrm{C} 11-\mathrm{C} 19-\mathrm{C} 24-\mathrm{C} 23 & -179.7(3)\end{array}$

Hydrogen-bond geometry $\left(\AA,{ }^{\circ}\right)$

\begin{tabular}{lllll}
\hline$D-\mathrm{H} \cdots A$ & $D-\mathrm{H}$ & $\mathrm{H} \cdots A$ & $D \cdots A$ & $D-\mathrm{H} \cdots A$ \\
\hline $\mathrm{C} 5-\mathrm{H} 2 \cdots \mathrm{Br}^{\mathrm{i}}$ & 0.95 & 2.84 & $3.528(4)$ & 130 \\
$\mathrm{C} 7-\mathrm{H} 4 \cdots \mathrm{Br}^{\mathrm{ii}}$ & 0.95 & 2.86 & $3.771(4)$ & 162 \\
$\mathrm{C} 12-\mathrm{H} 6 \cdots \mathrm{Br}^{\mathrm{iii}}$ & 0.95 & 2.75 & $3.688(7)$ & 171 \\
$\mathrm{C} 12-\mathrm{H} 6 \cdots \mathrm{O} 2^{\mathrm{iii}}$ & 0.95 & 2.55 & $3.491(7)$ & 173 \\
$\mathrm{C} 15-\mathrm{H} 7 \cdots \mathrm{Br} 2^{\mathrm{iii}}$ & 0.95 & 2.81 & $3.759(7)$ & 175 \\
$\mathrm{C} 15-\mathrm{H} 7 \cdots \mathrm{O} 2^{\mathrm{iii}}$ & 0.95 & 2.50 & $3.447(7)$ & 172 \\
$\mathrm{C} 16-\mathrm{H} 8 \cdots \mathrm{Br}^{\mathrm{ii}}$ & 0.95 & 2.52 & $3.286(7)$ & 138 \\
$\mathrm{C} 16-\mathrm{H} 8 \cdots \mathrm{O} 2^{\mathrm{ii}}$ & 0.95 & 2.57 & $3.363(7)$ & 141 \\
$\mathrm{C} 20-\mathrm{H} 11 \cdots \mathrm{Br} 1^{\mathrm{ii}}$ & 0.95 & 2.81 & $3.743(4)$ & 168 \\
$\mathrm{C} 20-\mathrm{H} 11 \cdots \mathrm{O} 3^{i \mathrm{ii}}$ & 0.95 & 2.55 & $3.446(18)$ & 158 \\
$\mathrm{C} 24-\mathrm{H} 15 \cdots \mathrm{Br}^{\mathrm{iii}}$ & 0.95 & 2.84 & $3.611(7)$ & 139 \\
\hline
\end{tabular}

Symmetry codes: (i) $-x+1,-y,-z+2$; (ii) $x,-y+1 / 2, z+1 / 2$; (iii) $-x, y+1 / 2,-z+3 / 2$; (iv) $x,-y+1 / 2, z-1 / 2$. 